\title{
PROPAGATION OF NON-STATIONARY CURVED AND STRETCHED PREMIXED FLAMES WITH MULTI-STEP REACTION MECHANISMS
}

\author{
A. Y. KLIMENKO* \\ Department of Mechanical Engineering, The University of Queensland, QId 4072, Australia \\ and
}

A. G. CLASS

Forschungzentrum Karlsruhe $\mathrm{GmbH}$, Institute for nuclear and energy technologies, D-76021 Karlsruhe, Germany

The propagation speed of a thin premixed flame disturbed by an unsteady fluid flow of a larger scale is considered. The flame may also have a general shape but the reaction zone is assumed to be thin compared to the flame thickness. Unlike in preceding publications, the presented asymptotic analysis is performed for a general, multi-step reaction mechanism and, at the same time, the flame front is curved by the fluid flow. The resulting equations define the propagation speed of disturbed flames in terms of the properties of undisturbed planar flames and the flame stretch. Special attention is paid to the near-equidiffusion limit. In this case, the flame propagation speed is shown to depend on the effective Zeldovich number $Z_{f}$, which is introduced in the paper, and the flame stretch. Unlike the conventional Zeldovich number, the effective Zeldovich number is not necessarily linked directly to the activation energies of the reactions. Several examples of determining the effective Zeldovich number for reduced combustion mechanisms are given in the paper while, for realistic reactions, the effective Zeldovich number is determined from experiments. Another feature of the present approach is represented by the relatively simple asymptotic technique based on the adaptive generalized curvilinear system of coordinates attached to the flame (i.e. IDFE - Intrinsic Disturbed Flame Equations).

Keywords: Flames premixed laminar; flames premixed turbulent; flame propagation velocity; flame stretch; flamelets; fluid dynamic aspects in combustion

\footnotetext{
${ }^{*}$ Corresponding author, full address

Dept. of Mechanical Engineering

The University of Queensland

Qld 4072, Australia

FAX: (61-7)-3365-4799

Email: klimenko@mech.uq.edu.au
} 


\section{Introduction}

Premixed combustion becomes increasingly important in applications that require advanced technology. Using premixed flames rather than diffusion flames enables the engineer to design engines that realize high energy densities. In general this reduces the cost of these engines, and the fluid or thermodynamic losses as well. However the fundamentals of diffusion flames are understood far better than those of premixed flames. The self-propagation mechanism of premixed flames is determined by a sophisticated balance of the chemical reaction, mass diffusion and convective transport of heat and species. In nonuniform time-dependent flows, strong interactions of the flow with this balance result in a non-constant local flame-propagation speed. In the present consideration, we study the interaction of a premixed flame with a general time-dependent flow, i.e. the flame is subject to strain, and the flame shape is both non-stationary and non-planar.

Since the flame is, typically, thin in comparison with the characteristic scales of the fluid flow (Bradley, Lau and Lawes 1992, Klimenko 1998), the most simple approach to premixed combustion is based on viewing the flame as a surface of discontinuity which separates the dense cold mixture from the light hot products. The flame behaves as a gas-dynamic discontinuity. A first model of this kind was proposed by Darrieus (1945) and independently by Landau (1944). In the Darrieus-Landau model, the flame is described as two incompressible fluids of different densities which are separated by the flame front. The model is completed by assuming that the flame propagates relative to the fluid at a fixed speed - the adiabatic flame speed. This heuristic assumption has proven to be oversimplified. It can easily be shown (Buckmaster and Ludford 1983) that planar flames described by the Darrieus-Landau model are unstable with respect to perturbations of any wave length. This result is in contradiction with the properties of planar flames which are known to exist in laboratory experiments. Obviously the flame speed depends on the geometry of the flame and possibly other effects. In order to overcome this deficiency of the Darrieus-Landau model, Markstein (1953) proposed a simple model, where the flame speed is assumed to be proportional to the flame curvature. The constant of proportionality is called the Markstein number. It should be noted that this model is empirical: the dependence of the flame speed on the flame curvature is postulated but not derived from the first principles. In the Markstein model, the short-wave perturbations are stabilized. In both the Darrieus-Landau and the Markstein model the details of the reaction mechanism are ignored. However if one aims to derive the dependence of the propagation speed of a flame on the geometry of the flame from first principles these details are essential.

Zeldovich, Barenblatt, Librovich and Makhviladze (1985) introduced a flame model, which ignores the gasdynamic effects but which takes into account the reaction kinetics. He assumed that the density of the fluid is constant, so that the flame does not effect the flow, but still the flow effects the flame. The kinetics was modeled as a one-step irreversible reaction between fuel and oxidizer. This reaction is deemed to have a high activation energy, the reaction rate strongly depends on the temperature. Otherwise, reaction would begin spontaneously in a combustible mixture. Due to the strong temperature dependence, the reaction zone is restricted to a thin zone near the highest temperature. Mathematically, this common property of the flames is expressed by a large value of the Zeldovich number $Z$. The thickness of the reaction zone can be assessed as $\sim l_{f} / Z$ where $l_{f}$ is the flame thickness. Behind the reaction zone, there is no reaction since all the available fuel is consumed in the reaction zone while, ahead of the reaction zone, the reaction rate is negligibly small due to the low temperature. The preheat zone is located ahead of the reaction zone. Within the preheat zone, the incoming mixture is preheated by heat conduction until reaction sets in at high temperatures. Also, within the preheat zone fuel diffuses towards the reaction zone where it is consumed. For hydrdocarbon/air flames at atmospheric conditions the thickness of the preheat zone is of the order of a tenth of a millimeter, i.e. much smaller than typical geometric length scales of a burner. At least for laminar flames variations across the flame are much stronger than those along the flame.

The interactions of the premixed flames formed by a one-step irreversible reaction with variable density fluid flows are considered in many publications (Sivashinsky 1976, Matalon and Matkowsky 1982, Bechtold and Matalon 1999) and reviewed by Clavin (1985). For these asymptotic models, Zeldovich's planar solution represents a zero-order approximation. At the next order of approximation, the corrections induced by the flame stretch are accounted for. The flame stretch $\kappa$ (Karlovitz, Denniston, Knapschaefer and Wells 1953) is defined as the differential increase of area of a flame surface segment and involves two terms: a term which is proportional to velocity gradients and another term which is proportional to the flame curvature (Matalon 1983, Williams 1985). The major asymptotic analyses of the influence of 
the flame stretch on the flame speed are performed by Sivashinsky (1976) and Matalon and Matkowsky (1982). They consider a thin flame formed by a one-step irreversible reaction with a high activation energy disturbed by an arbitrary external flow and assume that the flame curvature is weak as compared to the flame thickness. The flame characteristics depend on differential diffusion, i.e. the Lewis number $L$ which is defined as the ratio of heat diffusivity to mass diffusivity. Sivashinsky (1976) assumed that the Lewis number is bounded away from unity $\Delta L \equiv(L-1) \sim 1$ while Matalon and Matkowsky (1982) considered the case when the Lewis number is close to 1 so that $\Delta L \sim 1 / Z$. These formulations are different characteristic limits and result in different equations for the flame propagation speed. The analysis of the flames for the case of a near-stoichiometric mixture and a one-step reaction was performed only recently in Bechtold and Matalon (1999).

Realistic chemical mechanisms are, of course, much more complicated than a one-step irreversible reaction. The early works on sequential multistep reactions can be found in Kapila and Ludford (1977), Margolis and Matkowsky (1982) and on parallel reactions in Berman and Riazantsev (1973). Most asymptotic considerations of more realistic flames, involving multistep chemistry, are restricted to stationary planar situations. The reader is referred to Smooke (1991) for details. Typically there are several layers, where distinct reactions take place. Often the fuel is first broken into smaller molecules, i.e. into intermediate reactants. These intermediate reactants are further oxidized resulting in the final product. Reactions between highly reactive radicals are often a limiting step in the reaction mechanism. It is not our intention to discuss the details of these reaction mechanisms. However it should be noted that the lack of free radicals at low temperatures is responsible for the low reactivity of a combustible mixture at low temperatures. The reactions which generate or consume free radicals are often strongly temperature dependent. From the low reactivity of a combustible mixture at low temperatures, we may not conclude that a global single-step reaction modeling the combustion process necessarily has a high activation energy or Zeldovich number. This is only the case when a reaction with high activation energy is limiting.

There are several publications by Seshadri and Peters (1983), Rogg and Peters (1990), where the flame speeds of stationary planar stretched flames are considered for reduced multi-step reaction mechanisms. The authors take advantage of the fact, that for stagnation point flow the velocity component normal to the flame is independent of the transverse direction. This allows for one-dimensional solutions which are independent of transverse space. In stagnation point flow the flame stretch is proportional to the gradient of the normal velocity, which is a parameter in the one-dimensional equations. In Seshadri and Peters (1983), the reaction mechanism is represented by two reactions: a chain branching reaction and a chain braking reaction. The activation energy of the chain branching reaction is assumed to be large, while the activation energy of the chain braking reaction is zero. The chain branching reaction takes place in an inner layer which is thin compared to the preheat zone. This mechanism is similar to Zeldovich's two-step mechanism but both of the reactions considered by Seshadri and Peters (1983) have a significant heat release. Rogg and Peters (1990) analyzed the influence of the flame stretch on the three-step kinetic mechanism which was specifically designed for oxidation of methane.

In the present consideration, we do not restrict the analysis to a specific flow. We rather consider a time-dependent flame, which may be stretched and curved. This includes any geometry and any flow field as long as the curvature and the flame stretch do not exceed moderately large values. In the analysis we apply the approach based on the adaptive curvilinear system of coordinates which is attached to the flame (i.e. IDFE - the Intrinsic Disturbed Flame Equations obtained by Klimenko and Class (2000)), but now we consider a general reaction mechanism, although the reaction zone is presumed to be thin in comparison with the preheat zone. The most interesting aspect of the presented approach is that a certain integral characteristic of the undisturbed flames appears to be sufficient to determine the propagation speed of the flames curved and stretched by unsteady fluid flows. Even though there are certain cases, where the reaction zone is not thin as assumed in the present consideration, we want to emphasize the generic nature of our approach. In order to demonstrate efficiency of our method, we apply it to the one-step irreversible reaction mechanism and also to the reduced multistep mechanisms considered by Seshadri and Peters (1983) and Rogg and Peters (1990). Our results are consistent with the equations obtained in previous publications. We should note that, unlike in Seshadri and Peters (1983), our consideration of the Zeldovich two-step mechanism is of illustrative character and restricted only to the cases when complete analytical integration is possible. Our method can also applied to the realistic flames. In order to demonstrate this we use experimental results and some ideas presented in Abdel-Gayed, Bradley, Hamid and Lawes (1984) and Abdel-Gayed, Al-Khishali and Bradley (1984a).

The structure of the present manuscript is as follows. In section 2 the formulation of the governing 
equations is introduced. In section 3 the asymptotic analysis of the preheat and the reaction zone is presented including the matching of the layers to each other and to the outer flow. In section 4 the problem of an undisturbed flame is considered, which may be used as a reference solution, and allows us to omit to derive the full solution for all intermediate species. The general results are compared to the special limiting cases which have been studied by Sivashinsky (1976) and Matalon and Matkowsky (1982) in section 5. In section 6 and 7 the effective Zeldovich-numbers of flames with a simplified chemical kinetics and those with realistic chemical kinetics are determined. Here, the effective Zeldovich number represents an important undisturbed flame characteristic which enters the flame speed equation for disturbed flames. Finally, in section 8 we summarize our results.

\section{Formulation of the problem}

In the present work, we consider a general reaction mechanism whose integral effect can be symbolically represented by

$$
\nu_{\mathcal{A}} \mathcal{A}+\nu_{\mathcal{B}} \mathcal{B}+\left(\ldots+\nu_{\mathcal{R}_{i}} \mathcal{R}_{i}+\ldots\right) \rightarrow \nu_{\mathcal{P}_{1}} \mathcal{P}_{1}+\ldots+\nu_{\mathcal{P}_{j}} \mathcal{P}_{j}+\left(\ldots+\nu_{\mathcal{R}_{i}} \mathcal{R}_{i}+\ldots\right)+\text { heat }
$$

This reaction specifies conversion of the reactants $\mathcal{A}$ and $\mathcal{B}$ into one or more products $\mathcal{P}_{i}$. The reactant $\mathcal{A}$ is assumed to be deficient. The reaction is complete so that $\mathcal{A}$ is not present in the products (this assumption is, obviously, linked to the assumption of a thin reaction zone which is discussed below). The terms in the brackets involving $\mathcal{R}_{i}$ indicate the presence of a set of intermediate species which are generated and consumed in the intermediate reaction steps. The enthalpy, reactants and products can be called "the major species" to distinguish them from the intermediate species. The values of $\nu_{\mathcal{I}}$ represents the reaction coefficients of species denoted by $\mathcal{I}$. The system of equations governing transport, production and consumption of species for low Mach flows is represented by the conventional conservation of mass, conservation of energy and conservation of species equations.

$$
\begin{gathered}
\frac{\partial \rho}{\partial t}+\operatorname{div}(\rho \mathbf{v})=0 \\
\Lambda(1)[h]=W_{h} \\
\Lambda\left(L_{\mathcal{I}}\right)\left[C_{\mathcal{I}}\right]=W_{\mathcal{I}}
\end{gathered}
$$

where $h$ represents the sensible enthalpy and $\Lambda(L)[\ldots]$ denotes the convective operator

$$
\Lambda(L)[c] \equiv \rho \frac{\partial c}{\partial t}+\rho \mathbf{v} \cdot \nabla c-\operatorname{div}\left(\frac{D}{L} \nabla c\right) \quad \text { for any } c
$$

This operator depends on the Lewis number $L$. The "dynamic" diffusion coefficient is denoted here by $D$ is defined $D \equiv \tilde{D} \rho$ where $\tilde{D}$ is conventional "kinematic" diffusion coefficient. In the scalar transport equations given by (4), the binary mutual diffusion fluxes are conventionally replaced by the diffusion coefficients $D / L_{\mathcal{I}}$ implicitly assuming that the diffusion of the components occurs with respect to an inert gas $\mathcal{G}$ whose concentration is dominant in the mixture. In this case, the diffusion coefficients depend on temperature and do not depend on concentrations. The sensible enthalpy is, generally, specified by the integral

$$
h=h_{s}^{*}+\int_{T^{*}}^{T} c_{p} d T
$$

where $h_{s}^{*}$ is sensible enthalpy at $T=T^{*}$. Considering that the concentration of $\mathcal{G}$ is dominant, it is logical to assume that the heat capacity $c_{p}$ is determined by the heat capacity of $\mathcal{G}$ and $c_{p}=c_{p}(T)$ does not depend on concentrations. The heat released by the reactants is expected to be significant. In this case, $\nabla h=c_{p} \nabla T$ and $D(T)=k_{T} / c_{p}$ where $k_{T}$ is thermal conductivity. Another conventional assumption is given by $c_{p}=$ const which makes the sensible enthalpy proportional to temperature so that equation (3) can be written for $T$. If $c_{p}$ is not constant, then, as it is discussed above, $T$ and $h$ are linked by a function 
$h=h(T)$ (or $T=T(h)$ ) which is, generally, non-linear. Practically, the gas $\mathcal{G}$ is represented by molecular nitrogen whose heat capacity $c_{p}$ changes $\sim 25 \%$ as the temperature increases from $300 \mathrm{~K}$ to $3000 \mathrm{~K}$. Thus, it is more accurate to retain a general dependence $h=h(T)$ in the following analysis but the assumption $c_{p}=$ const is also acceptable.

The values of $W$ represent the influence of chemical reactions which are negligibly small everywhere with exception of the thin reaction zone where reactions are very intense. The actual equations for the reaction rates are, obviously, dependent on the reaction mechanism used. With exception of section 6 where specific examples are considered, we do not presume any particular form of the reaction rates. The major assumption of the present work which involves the characteristic scale of fluid motions $l_{m}$, the flame thickness $l_{f}$ and the thickness of the reaction zone $l_{r}$ is represented by the inequality

$$
l_{m} \gg l_{f} \gg l_{r}
$$

\section{The flame structure}

\subsection{The preheat zone}

In this section we consider the transport processes within the flame (whose characteristic scale is of order of $l_{f}$ ) disturbed by general unsteady fluid flow fluctuations of larger scale $l_{m}$. The influence of reaction terms is confined to a thin reaction zone $\left(\sim l_{r}\right)$. In the preheat zone, this influence can be neglected and the flame structure is determined by diffusion and convection

$$
\Lambda(1)[h]=0, \quad \Lambda\left(L_{\mathcal{I}}\right)\left[C_{\mathcal{I}}\right]=0
$$

where $\mathcal{I}$ denotes any of the species. The boundary conditions for the major species are given by

$$
h \rightarrow h_{u}, C_{\mathcal{A}} \rightarrow C_{\mathcal{A} u}, C_{\mathcal{B}} \rightarrow C_{\mathcal{B} u}, C_{\mathcal{P}_{i}} \rightarrow C_{\mathcal{P}_{i} u}, C_{\mathcal{R}_{i}} \rightarrow 0 \text { as } x^{1} \rightarrow-\infty \text { (unburned mixture) }
$$

The subscript " $u$ " denotes values of the parameters in the unburned mixture while the subscript " $b$ " is used to denote those values related to the burned mixture. Typically, the products $\mathcal{P}_{i}$ are not present in the unburned mixture such that $C_{\mathcal{P}_{i} u}=0$. However it is possible that some amounts of the products are premixed with reactants before igniting the flame (for example water vapor or recirculated flue gases). The unburned values of the concentrations and the enthalpy are prescribed by the experimental conditions.

The preheat zone is the widest zone of the flame and, therefore, this zone is primarily affected by the fluid flow. We follow our previous work (Klimenko and Class 2000) and select a moving generalized curvilinear system of coordinates $x^{1}, x^{2}, x^{3}$ so that $x^{1}=0$ specifies the location of the reaction zones. With these coordinates, the conservation equation of mass and the convective operator take the form

$$
\begin{gathered}
\frac{\partial g^{1 / 2} \rho}{\partial t}+\frac{\partial g^{1 / 2} m^{i}}{\partial x^{i}}=0 \\
\Lambda(L)[c]=\rho \frac{\partial c}{\partial t}+m^{i} \frac{\partial c}{\partial x^{i}}-\frac{1}{g^{1 / 2}} \frac{\partial}{\partial x^{j}}\left(g^{i j} g^{1 / 2} \frac{D}{L} \frac{\partial c}{\partial x^{i}}\right)
\end{gathered}
$$

where $g^{i j}$ is the metric tensor; $g \equiv \operatorname{det}\left(g_{i j}\right)=1 / \operatorname{det}\left(g^{i j}\right) ; m^{i} \equiv \rho U^{i} ; i, j=1,2,3$ and the sum is taken over repeated indices. The contravariant components $U^{i}$ specify the fluid velocity relative to the moving system of coordinates $U^{i} \equiv v^{i}+d x^{i} / d t$ where $d x^{i} / d t$ is evaluated for constant values of the Cartesian coordinates.

The derivation of IDFE requires several steps which are fully presented in Klimenko and Class (2000). The major steps of this approach involve selecting the coordinate $x^{1}$ to be normal to the flame surface. The orthogonality condition requires that $g^{1 \alpha}=g^{\alpha 1}=g_{1 \alpha}=g_{\alpha 1}=0$ where the Greek indices run over the flame surface coordinates: $\alpha=2,3$. We assume that, in the vicinity of the point of consideration, $g^{11}=g_{11}=1$, and thus the coordinate $x^{1}$ represents the physical coordinate normal to the flame. Alternative assumptions would result in more complicated equations involving additional geometrical terms which should not affect (and do not affect) the final equations (Klimenko and Class 2000). Since $\operatorname{det}\left(g_{\alpha \beta}\right)=g / g_{11}=g$, the value of $g$ specifies the element of the flame surface area. 
We introduce stretched coordinates $X^{i}=x^{i} / \varepsilon$ and $\tau=t / \varepsilon$ which characterize the outer fluid flow. Here, the small parameter $\varepsilon$ represents the ratio $\varepsilon \equiv l_{f} / l_{m}$. The physical coordinates $x^{i}$ are assumed to be of unity order within the flame. Since $g$ and other geometrical parameters are determined by the outer flow, they must depend on the stretched coordinates while other parameters, such as the enthalpy (or the temperature) and the concentrations change within the flame and depend on inner variables. Without loss of generality, the coordinates on the flame surface are selected so that $U^{\alpha}=0$ to the leading order of the asymptotic analysis (i.e. $d x^{\alpha} / d t=-v^{\alpha}$ on the flame surface, $\alpha=2,3$ ). The leading terms of equations (10) and (11) are given by

$$
\begin{gathered}
\varepsilon \frac{\partial g_{f}^{1 / 2} \rho}{\partial \tau}+\varepsilon\left(\frac{\partial g^{1 / 2}}{\partial X^{1}}\right)_{f} m^{1}+\left(g_{f}^{1 / 2}+\varepsilon\left(\frac{\partial g^{1 / 2}}{\partial X^{1}}\right)_{f} x^{1}\right) \frac{\partial m^{1}}{\partial x^{1}}+\ldots=0 \\
\Lambda(L)[c]=\varepsilon \rho \frac{\partial c}{\partial \tau}+m^{1} \frac{\partial c}{\partial x^{1}}-\frac{\partial}{\partial x^{1}}\left(\frac{D}{L} \frac{\partial c}{\partial x^{1}}\right)-\varepsilon \frac{D}{L}\left(\frac{\partial \ln \left(g^{1 / 2}\right)}{\partial X^{1}}\right)_{f} \frac{\partial c}{\partial x^{1}}+\ldots
\end{gathered}
$$

Terms of higher order, which are not needed in the present analysis, are omitted. The subscript " $f$ " indicates that the indexed value is either taken at the flame surface, is related to whole flame structure, or remains constant within the flame. Further in the paper, we denote $m \equiv m^{1}, x \equiv x^{1}$, etc. for convenience. Using the rescaled flame stretch $K_{f}$ and the flame curvature $\Gamma_{f}$ which are defined by

$$
K_{f} \equiv\left(\frac{\partial \ln \left(G^{1 / 2}\right)}{\partial \tau}\right)_{f}, \quad \Gamma_{f} \equiv-\left(\frac{\partial \ln \left(G^{1 / 2}\right)}{\partial X}\right)_{f}
$$

and linked to the physical flame stretch $\kappa_{f}$ and to the physical flame curvature $\gamma_{f}$ by $\kappa_{f}=\varepsilon K_{f}$ and $\gamma_{f}=\varepsilon \Gamma_{f}$, we rewrite the IDFE in the form

$$
\begin{gathered}
\varepsilon K_{f} \rho+\varepsilon \frac{\partial \rho}{\partial \tau}-\varepsilon \Gamma_{f} m+\left(1-\varepsilon \Gamma_{f} x\right) \frac{\partial m}{\partial x}+\ldots=0 \\
\Lambda(L)[c]=\varepsilon \rho \frac{\partial c}{\partial \tau}+m \frac{\partial c}{\partial x}-\frac{\partial}{\partial x}\left(\frac{D}{L} \frac{\partial c}{\partial x}\right)+\varepsilon \frac{D}{L} \Gamma_{f} \frac{\partial c}{\partial x}+\ldots
\end{gathered}
$$

The major feature of these equations is that IDFE explicitly involve two major parameters of the flame/flow interactions: the flame stretch $K_{f}$ and the flame curvature $\Gamma_{f}$. IDFE are relatively simple and can be used to find efficient solutions for variety of problems. The sign of $\Gamma_{f}$ takes into account that the flame propagates towards $x=-\infty$. It is convenient to rewrite equation (16) in terms of the normal flux operator $S$ defined by $S(L)[c] \equiv m c-(D / L)(\partial c / \partial x)$

$$
\Lambda(L)[c]=\frac{\partial S(L)[c]}{\partial x}-\varepsilon \Gamma_{f} S(L)[c]+\varepsilon \frac{\partial c \rho}{\partial \tau}+\varepsilon K_{f} c \rho+. .
$$

The mass flow rate and the other variables are represented by the expansions $m=m_{0}+\varepsilon m_{1}+\ldots$, $c=c_{0}+\varepsilon c_{1}+. ., h=h_{0}+\varepsilon h_{1}+\ldots, \rho=\rho_{0}+\varepsilon \rho_{1}+\ldots, D=D_{0}+\varepsilon D_{1}+\ldots, C_{\mathcal{I}}=\left(C_{\mathcal{I}}\right)_{0}+\varepsilon\left(C_{\mathcal{I}}\right)_{1}+\ldots$ Here, we analyze equation (16) using a function $c(x, L)$ which satisfies $\Lambda(L)[c]=0$ subject to the boundary conditions $c \rightarrow 0$ as $x \rightarrow-\infty$. The solution of equation (15) is

$$
m_{0}=\text { const }, \quad m_{1}(x)=\int_{x}^{0} \Psi_{m} d x+m_{1 b}, \quad \Psi_{m} \equiv \rho_{0} K_{f}-m_{0} \Gamma_{f}+\frac{\partial \rho_{0}}{\partial \tau}
$$

while the solution of equation (16) takes the form

$$
\begin{gathered}
c_{0}(x, L)=\exp \left(m_{0} \int_{0}^{x} L \frac{d x}{D_{0}}\right) \\
c_{1}(x, L)=\frac{c_{0}(x, L)}{m_{0}} \int_{x}^{0} \frac{\Psi_{c}(x, L)}{c_{0}(x, L)} d x+\frac{1}{m_{0}} \int_{-\infty}^{x} \Psi_{c}(x, L) d x
\end{gathered}
$$




$$
\Psi_{c}(x, L) \equiv-\frac{\partial m_{1} c_{0}}{\partial x}-K_{f} c_{0} \rho_{0}-\frac{\partial c_{0} \rho_{0}}{\partial \tau}+\frac{\partial}{\partial x}\left(\frac{D_{1}}{L} \frac{\partial c_{0}}{\partial x}\right)
$$

so that

$$
c(0, L)=1+\varepsilon \Psi_{r}(L)+O\left(\varepsilon^{2}\right), \quad c^{\prime}(0, L)=\left(\frac{L}{D_{0}}\right)_{b} m_{0}+O\left(\varepsilon^{2}\right)
$$

where

$$
\begin{gathered}
c^{\prime} \equiv \frac{\partial c}{\partial x}, \quad \Psi_{r}(L) \equiv \frac{1}{m_{0}} \int_{-\infty}^{0} \Psi_{c}(x, L) d x=-\frac{m_{1 b}}{m_{0}}+\frac{D_{1 b}}{D_{0 b}}-\Phi(L) \\
\Phi(L) \equiv \frac{1}{m_{0}}\left(K_{f}+\frac{d}{d \tau}\right)[\phi(L)], \quad \phi(L) \equiv \int_{-\infty}^{x} \rho_{0} c_{0}(x, L) d x
\end{gathered}
$$

The enthalpy and concentrations of the components are governed by the equations

$$
h=h_{u}+a_{h} c(x, 1), \quad C_{\mathcal{I}}=C_{\mathcal{I} u}+a_{\mathcal{I}} c\left(x, L_{\mathcal{I}}\right)
$$

which take into account the conditions in the unburned fluid. The coefficients $a$ are to be determined by matching to the reaction zone. Meanwhile we obtain the values of the parameters and their derivatives as $x \rightarrow-0$

$$
\begin{gathered}
(h)_{r}=h_{u}+a_{h}\left(1+\varepsilon \Psi_{r}(1)\right)+O\left(\varepsilon^{2}\right), \quad\left(C_{\mathcal{I}}\right)_{r}=C_{\mathcal{I} u}+a_{\mathcal{I}}\left(1+\varepsilon \Psi_{r}\left(L_{\mathcal{I}}\right)\right)+O\left(\varepsilon^{2}\right) \\
\left(\frac{\partial h}{\partial x}\right)_{r}=a_{h} \frac{m_{0}}{D_{0 b}}+O\left(\varepsilon^{2}\right), \quad\left(\frac{\partial C_{\mathcal{I}}}{\partial x}\right)_{r}=a_{\mathcal{I}}\left(\frac{L_{\mathcal{I}}}{D_{0}}\right)_{b} m_{0}+O\left(\varepsilon^{2}\right)
\end{gathered}
$$

Here the subscript index " $r$ " denotes the values related to the location $x \rightarrow-0$ (i.e. just before the reaction zone which is assumed to be at $x=0)$. The subscript " $b$ " indicates values evaluated in the burned mixture. Note that for some parameters, the subscript " $r$ " has the same meaning as " $b "$. As determined by the reactions zone analysis and asymptotic matching presented in the following sections, these indexed values are identical to the order of precision considered in the present analysis. For other parameters, the meanings of " $r$ " and " $b "$ are different. For example $(h)_{r}=h_{b}$ and $D_{r}=D_{b}$ but $(\partial h / \partial x)_{r} \neq(\partial h / \partial x)_{b}$ since $(\partial h / \partial x)_{b}=0$.

\subsection{The reaction zone}

In this section we consider the reaction zone, where the combustion reactions take place. The major assumption which is used in the present analysis is the requirement of a thin reaction zone. Here thin means that the thickness of the reaction zone is much smaller than the thickness of the preheat zone. Therefore, we introduce a small parameter $\varepsilon_{r}=l_{r} / l_{f}$. The characteristic variable of the reaction zone is denoted by $\xi=x / \varepsilon_{r}$. First, we apply the asymptotic analysis of the reaction zone to the generic equation $\Lambda(L)[C]=W$. The symbolic variables are represented by the expansions $C=C_{b}+\varepsilon_{r} C_{1}+\ldots$, $W=\varepsilon_{r}^{-1} W_{0}+\ldots$, etc. At the leading order of an asymptotic series expansion in powers of $\varepsilon_{r}$ the convective terms do not contribute and the generic equation is given by

$$
-\left(\frac{D}{L}\right)_{b} \frac{\partial^{2} C_{1}}{\partial \xi^{2}}=W_{0}
$$

Integrating the generic equation across the reaction zone yields

$$
\left(\frac{D}{L}\right)_{b}\left(\frac{\partial C}{\partial \xi}\right)_{r}=\int_{-\infty}^{+\infty} W_{0} d \xi
$$


where the subscript " $r$ " denotes the intermediate limit specified by $\xi \rightarrow-\infty, x \rightarrow-0$ which corresponds to the location just before the reaction zone. We transform these equations back to the original coordinate $x$ when applying them to the enthalpy and other species

$$
-D_{b} \frac{\partial^{2} h}{\partial x^{2}}=W_{h}, \quad-\left(\frac{D}{L_{\mathcal{I}}}\right)_{b} \frac{\partial^{2} C_{\mathcal{I}}}{\partial x^{2}}=W_{\mathcal{I}}
$$

where "I " denotes any of the species. Note that, to the order of $O\left(\varepsilon_{r} \varepsilon\right)$, the reaction zone remains plane and undisturbed. The integral of the reaction rate over the reaction zone specifies the integral rate of consumption (or production) of a particular reactant. Thus, the integral rates of consumption of reactants and generation of products are linked by the reaction coefficients while the overall rate of generation of the intermediate species is zero (i.e., for the intermediate species, the volume average of the consumption rate is the same as the volume average of the generation rate). Evaluation of the integral (29) for different species yields

$$
\begin{gathered}
\left(\frac{D}{L_{\mathcal{A}}}\right)_{b}\left(\frac{\partial C_{\mathcal{A}}}{\partial x}\right)_{r}=-M_{\mathcal{A}}=\int_{-\infty}^{+\infty} W_{\mathcal{A}} d x \\
D_{b}\left(\frac{\partial h}{\partial x}\right)_{r}=N_{h} M_{\mathcal{A}}, \quad\left(\frac{D}{L_{\mathcal{B}}}\right)_{b}\left(\frac{\partial C_{\mathcal{B}}}{\partial x}\right)_{r}=N_{\mathcal{B}} M_{\mathcal{A}} \\
\left(\frac{D}{L_{\mathcal{P}_{i}}}\right)_{b}\left(\frac{\partial C_{\mathcal{P}_{i}}}{\partial x}\right)_{r}=N_{\mathcal{P}_{i}} M_{\mathcal{A}}, \quad\left(\frac{\partial C_{\mathcal{R}_{i}}}{\partial x}\right)_{r}=0
\end{gathered}
$$

where the value $M_{\mathcal{A}}$ is the mass rate of consumption of the deficient reactant in the reaction zone. The values $N_{\mathcal{I}}= \pm\left(\nu_{\mathcal{I}} w_{\mathcal{I}}\right) /\left(\nu_{\mathcal{A}} w_{\mathcal{A}}\right)$ are introduced for the major chemical species and the positive sign is selected for products (and the sensible enthalpy) while the negative sign is selected for the reactants. Obviously, $N_{\mathcal{A}}=-1$. Here $\nu_{\mathcal{I}}$ denote the reaction coefficients and the $w_{\mathcal{I}}$ denote the molecular weights of the substances $\mathcal{I}$. The value $N_{h}$ represents the heat released when burning one unit mass of the deficient reactant. The values $N_{\mathcal{I}}$ and $N_{h}$ remain constant (as long as the reaction mechanism is not changed). Although $N_{h}$ is presumed constant, the adiabatic temperature $T_{0 b}=T\left(h_{0 b}\right)$, where $h_{0 b}=h_{u}+N_{h} C_{\mathcal{A} u}$, can be changed by altering $C_{\mathcal{A} u}$ - the amount of the deficient reactant present in the fresh mixture, or by changing the temperature $T_{u}$ and the enthalpy $h_{u}=h_{u}\left(T_{u}\right)$ of the fresh mixture.

Assuming that the values of the enthalpy (or temperature) and concentrations in the burned mixture, which are indexed by the subscript " $b$ ", are given, the solution of the problem (30)-(31) specifies the consumption rate of the deficient reactants as the function of the other parameters of the reaction zone

$$
M_{\mathcal{A}}=F_{\mathcal{A}}\left(h_{b}, C_{\mathcal{I} b}, N_{h}, N_{\mathcal{I}}, D, L_{\mathcal{I}}, \rho\right)=F_{\mathcal{A}}\left(T_{b}, C_{\mathcal{B} b}, C_{\mathcal{P}_{i} b}\right)
$$

We will call the function $F_{\mathcal{A}}$ the consumption rate function. In this equation, the burned concentrations of the deficient reactant $\mathcal{A}$ and of the intermediate species $\mathcal{R}_{i}$ are zeros and should be excluded from the function $F_{\mathcal{A}}$. We also omit the values $N_{\mathcal{I}}$ and $N_{h}$, which can not be altered as long as fixed reaction mechanisms and complete combustion are considered. The sensible enthalpy $h$, the density $\rho$, and the diffusion coefficient $D$ (and possibly the Lewis numbers if the $L_{\mathcal{I}}$ are not constant) are presumed to be functions of the temperature. The corresponding values are determined by $T_{b}$. The dependence of $M_{\mathcal{A}}$ on $T_{b}$ involves these functions. Although those parameters, which are not expected to change, are excluded from the list of parameters on the right-hand side of equation (32), the actual values of the parameters in the burned mixture, which are indexed with the subscript " $b$ " should be determined by matching with the preheat zone.

\subsection{Matching}

First we notice that, as it follows from equations (27) and (31), $a_{\mathcal{R}_{i}}=0$ for intermediate species. Hence the reaction zone boundary conditions for intermediate species take the form

$$
\left(C_{\mathcal{R}_{i}}\right)_{r}=0+O\left(\varepsilon^{2}\right), \quad\left(C_{\mathcal{R}_{i}}\right)_{b}=0+O\left(\varepsilon^{2}\right)
$$


If the reaction zone is thin, (as assumed in our analysis) the intermediate species are, to the asymptotic precision needed in our analysis, confined to the reaction zone. The boundary conditions for the major species need a more detailed inspection. Comparison of equations (27) and (31) indicates that

$$
a_{h}=\frac{M}{m_{0}} \frac{D_{0 b}}{D_{b}} N_{h} C_{\mathcal{A} u}+O\left(\varepsilon^{2}\right), \quad a_{\mathcal{I}}=\frac{M}{m_{0}} \frac{D_{0 b}}{D_{b}} N_{\mathcal{I}} C_{\mathcal{A} u}+O\left(\varepsilon^{2}\right), \quad M \equiv \frac{M_{\mathcal{A}}}{C_{\mathcal{A} u}}
$$

where a new parameter, denoted as $M$ and having the same dimension as $m$, is introduced. Equations (26) specify, to the order of the matching procedure, the values of the enthalpy and concentrations in the burned mixture

$$
\begin{gathered}
h_{b}=h_{u}+N_{h} C_{\mathcal{A} u} \frac{M}{m_{0}}\left(1-\varepsilon \frac{m_{1 r}}{m_{0}}-\varepsilon \Phi(1)\right)+O\left(\varepsilon^{2}\right) \\
C_{\mathcal{I} b}=C_{\mathcal{I} u}+N_{\mathcal{I}} C_{\mathcal{A} u} \frac{M}{m_{0}}\left(1-\varepsilon \frac{m_{1 r}}{m_{0}}-\varepsilon \Phi\left(L_{\mathcal{I}}\right)\right)+O\left(\varepsilon^{2}\right)
\end{gathered}
$$

Here we use equation (23) and expand $D_{b}$ into the series $D_{b}=D_{0 b}+\varepsilon D_{1 b}+\ldots$ The terms of order $\varepsilon^{2}$ and higher are consistently neglected. The subscript "I $\mathcal{I}$ " denotes " $\mathcal{A}$ ", " $\mathcal{B}$ " or " $\mathcal{P} i$ ".

Since $\mathcal{A}$ is the deficient reactant and the reactions are complete, this reactant is not present in the products: $\left(C_{\mathcal{A}}\right)_{b}=0$. We note that $N_{\mathcal{A}}=-1$, substitute $\left(C_{\mathcal{A}}\right)_{b}=0$ into $(36)$ and obtain

$$
\frac{M}{m_{0}}=1+\varepsilon \frac{m_{1 r}}{m_{0}}+\varepsilon \Phi\left(L_{\mathcal{A}}\right)+O\left(\varepsilon^{2}\right)
$$

This equation demonstrates that $M$ should be represented by the expansion $M=M_{0}+\varepsilon M_{1}+\ldots$ so that $M_{0}=m_{0}$ and $M_{1}=m_{0} \Phi\left(L_{\mathcal{A}}\right)+m_{1 r}$. Substitution of $M$ specified by (37) into (35) and (36) while taking into account (24) yields

$$
\begin{gathered}
h_{b}=h_{u}+(\Delta h)_{0}+\varepsilon(\Delta h)_{1}+O\left(\varepsilon^{2}\right) \\
(\Delta h)_{0} \equiv N_{h} C_{\mathcal{A} u}, \quad(\Delta h)_{1} \equiv \frac{(\Delta h)_{0}}{m_{0}}\left(K_{f}+\frac{d}{d \tau}\right)\left[\phi\left(L_{\mathcal{A}}\right)-\phi(1)\right] \\
C_{\mathcal{I} b}=C_{\mathcal{I} u}+\left(\Delta C_{\mathcal{I}}\right)_{0}+\varepsilon\left(\Delta C_{\mathcal{I}}\right)_{1}+O\left(\varepsilon^{2}\right) \\
\left(\Delta C_{\mathcal{I}}\right)_{0} \equiv N_{\mathcal{I}} C_{\mathcal{A} u}, \quad\left(\Delta C_{\mathcal{I}}\right)_{1} \equiv \frac{\left(\Delta C_{\mathcal{I}}\right)_{0}}{m_{0}}\left(K_{f}+\frac{d}{d \tau}\right)\left[\phi\left(L_{\mathcal{A}}\right)-\phi\left(L_{\mathcal{I}}\right)\right]
\end{gathered}
$$

where the index " $\mathcal{I}$ " represents any of the following species " $\mathcal{P}_{i}$ " or " $\mathcal{B}$ ". If " $\mathcal{I}$ " is " $\mathcal{A}$ " then, obviously, $\left(C_{\mathcal{A}}\right)_{b}=0$. The values $(\Delta \ldots)_{0}$ specify jumps of the parameters in undisturbed flames (one can formally put $\varepsilon=0$ in (38) and (39) to find this) and these values are positive for the sensible enthalpy and products and negative for reactants. The equations (38) and (39) in conjunction with the equations (33) and (31) define the boundary conditions for the reaction zone equations (30).

Presuming that the value of $M=F_{\mathcal{A}}(\ldots) / C_{\mathcal{A} u}$ is known (i.e. obtained by solving the reaction zone equations), we can now determine the propagation speed of the flame $U_{f}$ which is conventionally defined as $U_{f}=m_{u r} / \rho_{u}$. Here, $m_{u r}$ denotes $m_{u}$ taken at the location of the reaction zone (i.e. at $x=0$ ) while $m_{u}$ represents the unburned mass flow rate which is evaluated under the assumption that the density does not changed within the flame (i.e. $m_{u}$ is an extension of the unburned mass flow rate into the flame). The actual mass flow rate $m$ is governed by equation (15) while the unburned mass flow rate $m_{u}$ satisfies the equation (15) with $\rho=\rho_{u}$. Thus, we have

$$
m_{u r}=m_{r}-\varepsilon\left(K_{f}+\frac{\partial}{\partial \tau}\right)\left[\phi_{\rho}\right]+O\left(\varepsilon^{2}\right), \quad \phi_{\rho} \equiv \int_{-\infty}^{0}\left(\rho_{u}-\rho_{0}\right) d x
$$


Equations (24), (37), (40) and the expansion $m_{r}=m_{0}+\varepsilon m_{1 r}+\ldots$ determine the following equation for the unburned mass flow rate evaluated at $x=0$

$$
m_{u r}=M-\varepsilon\left(K_{f}+\frac{\partial}{\partial \tau}\right)\left[\phi\left(L_{\mathcal{A}}\right)+\phi_{\rho}\right]+O\left(\varepsilon^{2}\right), \quad U_{f}=m_{u r} / \rho_{u}, \quad M=\frac{F_{\mathcal{A}}(\ldots)}{C_{\mathcal{A} u}}
$$

The equations obtained here are suitable for the analysis of different asymptotic limits, as determined by the properties of the function $F_{\mathcal{A}}(\ldots)$. Note that the value of $m_{0}$ does not necessarily represent the value of mass flow rate in a planar undisturbed flame $m_{*}$ (some examples of $m_{0} \neq m_{*}$ are given in the following sections). We use the subscript "*" to denote values related to the undisturbed flames under similar conditions. However, $(\ldots)_{0}=(\ldots)_{*}$ appears to be correct for some values. The value $(\Delta h)_{0} \equiv N_{h} C_{\mathcal{A} u}=(\Delta h)_{*}$ is not affected when disturbing the flame so that $h_{0 b}=h_{b *}, T_{0 b}=T_{b *}$ etc. Note that this equality does not, apply to the whole enthalpy and temperature profiles: $h_{0} \neq h_{*}$ and $T_{0} \neq T_{*}$. In the rest of the paper, we use the "asterisk notation" whenever suitable to emphasize the invariable nature of some parameters.

The integrals $\phi$ and $\phi_{\rho}$ can be written in a more convenient form if the sensible enthalpy, density and the diffusion coefficient are functions of the temperature only $h_{0}=h\left(T_{0}\right), \rho_{0}=\rho\left(T_{0}\right)$, and $D=D\left(T_{0}\right)$. Equations (19), (25), (34), and (38) indicate $h_{0}=h_{u}+(\Delta h)_{*} c_{0}(x, 1)$ so that $c_{p} d T_{0}=d h_{0}=(\Delta h)_{*} d c_{0}$ where $d c_{0}=c_{0} m_{0} D_{0}^{-1} d x$. By replacing integration over $x$ by integration over $T$, the definitions of $\phi$ and $\phi_{\rho}$ in (24) and (40) are transformed into

$$
\begin{gathered}
\phi(L)=m_{0} \frac{\tilde{D}_{u}}{U_{f 0}^{2}} I(L), \quad I(L) \equiv \frac{1}{(\Delta h)_{*}^{L}} \int_{T_{u}}^{T_{b *}} \hat{\rho}_{0} \hat{D}_{0}\left(h_{0}-h_{u}\right)^{L-1} c_{p} d T_{0} \\
\phi_{\rho}=m_{0} \frac{\tilde{D}_{u}}{U_{f 0}^{2}} I_{\rho}, \quad I_{\rho} \equiv \int_{T_{u}}^{T_{b *}} \hat{D}_{0} \frac{1-\hat{\rho}_{0}}{h_{0}-h_{u}} c_{p} d T_{0}
\end{gathered}
$$

where we use the following notations

$$
U_{f 0} \equiv \frac{m_{0}}{\rho_{u}}, \quad \tilde{D}_{u} \equiv \frac{D_{u}}{\rho_{u}}, \quad \hat{\rho}_{0} \equiv \frac{\rho_{0}}{\rho_{u}}, \quad \hat{D}_{0} \equiv \frac{D_{0}}{D_{u}}, \quad(\Delta h)_{*} \equiv N_{h} C_{\mathcal{A} u}=h_{b *}-h_{u}
$$

Here, $h_{b *}$ is the sensible enthalpy of the burned mixture of an undisturbed flame (which is linked to the adiabatic temperature $T_{b *}=T\left(h_{b *}\right)$ ). Generally, the value of the Lewis numbers which represent the ratio of thermal conductivity and diffusivity may depend on the temperature $L=L(T)$. In this case equation (42) specifies the integral $I(L)=I([L(T)])$ which appears to be a functional depending on the function $L(T)$. In practical applications, the values of the Lewis numbers vary much less with the temperature than the values of the diffusion coefficients and it is reasonable to treat them as constants resulting in a significant simplification of the integral $I$.

\section{Equivalent formulation for undisturbed flames}

In this section we formulate the problem for a planar stationary flame, using the consumption rate function $F_{\mathcal{A}}(\ldots)$ introduced in the previous section. Expanding the functional about the planar state will allow us to find a generic flame speed relation for perturbed flames. The consumption rate function $F_{\mathcal{A}}(\ldots)$ can be found by solving the problem, defined by $(30),(31),(38),(39)$ and (33), within the reaction zone and then determining the consumption rate of the deficient reactant $M_{\mathcal{A}}$. This approach is suitable when the reaction mechanism under consideration is not complicated and an analytical solution of the problem is sought. For more complicated kinetics, it could be more convenient to find structure of the undisturbed plane flame and determine the mass flow rate $m_{*}$. Solving the undisturbed equations

$$
\Lambda_{0}(1)[h]=W_{h}, \quad \Lambda_{0}\left(L_{\mathcal{I}}\right)\left[C_{\mathcal{I}}\right]=W_{\mathcal{I}}, \quad \Lambda_{0}(L)[c] \equiv m_{*} \frac{\partial c}{\partial x}-\frac{\partial}{\partial x}\left(\frac{D}{L} \frac{\partial c}{\partial x}\right)
$$

with conditions (9) in the unburned mixture is conventional. This solution determines $m_{*}=\rho_{u} U_{f *}$, which, as it follows from (37), coincides with $M$ in undisturbed flames (i.e. the physical meaning of the 
value $M$ introduced earlier is given by $M=m_{*}$ ). We use the subscript "*" to denote values related to an undisturbed flame. Generally, $M=m_{*}$ is a characteristics of the flame but not a characteristics of the reaction zone: the convective terms are neglected in the reaction zone. The reaction zone is characterized by the mass consumption rate of the deficient reactant $M_{\mathcal{A}}$ which, however, is easily determined from $M$ using $M_{\mathcal{A}}=m_{*} C_{\mathcal{A} u}$. The determined value of $M_{\mathcal{A}}$ may be expressed as a function of the parameters listed in equation (32): $M_{\mathcal{A}}=F_{\mathcal{A}}\left(T_{b}, C_{\mathcal{B} b}, C_{\mathcal{P}_{i} b}\right)$. This function implies dependence on other parameters (such as $N_{h}$ ) which are deemed to be constant and are not listed explicitly. Since the reaction zone of a disturbed flame, to the order of present analysis, is not disturbed by the flow, the function $F_{\mathcal{A}}(\ldots)$ is the same for both cases (disturbed and undisturbed) and can be used to determine the propagation speed of the disturbed flame. It should be noted that $M_{\mathcal{A}} /\left(\rho_{u} C_{\mathcal{A} u}\right)$ is the propagation speed of the undisturbed flame $U_{f *}$ while the propagation speed of the disturbed flame $U_{f}$ is, generally, different and determined by equation (41).

The previously obtained results can be summarized in the following proposition

Proposition 1 If the mass consumption rate of the deficient reactant of an undisturbed and plane premixed flame with a multistep combustion process occurring in a thin reaction zone is known and expressed as a function of values of the parameters in the burned mixture $M_{\mathcal{A}}=F_{\mathcal{A}}\left(T_{b}, C_{\mathcal{B} b}, C_{\mathcal{P}_{i} b}\right)$ (and, may be, some other parameters which are to be kept constant), then the propagation speed of the same flame disturbed by an arbitrary unsteady flow of a larger scale can be determined and is given by equation (41) with the arguments of the function $F_{\mathcal{A}}$ specified by equations (38), (39) and $T_{b}=T_{b}\left(h_{b}\right)$.

The equations mentioned in the proposition involve the integrals $\phi(L)$ and $\phi_{\rho}$ which are evaluated in (42) and (43). The procedure specified in the proposition allows for a simple physical interpretation: we consider both the disturbed flame and the undisturbed flame and, in order to provide similarity of the reaction zones and the values of $M_{\mathcal{A}}$, we require that the values of the parameters in the burned mixture of both flames should stay the same. One can view this as a replacement of the conditions in the unburned mixture (9) of an undisturbed flame by the effective values

$$
\begin{gathered}
h_{u}^{\circ}=h_{u}+\varepsilon(\Delta h)_{1}+N_{h}\left(C_{\mathcal{A} u}-C_{\mathcal{A} u}^{\circ}\right), \quad C_{\mathcal{B} u}^{\circ}=C_{\mathcal{B} u}+\varepsilon\left(\Delta C_{\mathcal{B}}\right)_{1}+N_{\mathcal{B}}\left(C_{\mathcal{A} u}-C_{\mathcal{A} u}^{\circ}\right) \\
C_{\mathcal{P}_{i} u}^{\circ}=C_{\mathcal{P}_{i} u}+\varepsilon\left(\Delta C_{\mathcal{P}_{i}}\right)_{1}+N_{\mathcal{P}_{i}}\left(C_{\mathcal{A} u}-C_{\mathcal{A}_{u}}^{\circ}\right), \quad C_{\mathcal{R}_{i} u}^{\circ}=0
\end{gathered}
$$

The values $(\Delta \ldots)_{1}$ are defined in (38) and (39). The values of $C_{\mathcal{A} u}^{\circ}$ are taken the same as $C_{\mathcal{A} u}$ or slightly altered $\left(\left(C_{\mathcal{A} u}-C_{\mathcal{A} u}^{\circ}\right) \sim \varepsilon\right)$ to ensure positiveness of all concentrations in (45), although the reactant $\mathcal{A}$ should still remain deficient. The effective conditions marked by the subscript "॰" provide that, to the order required in the present analysis, the conditions in the burned mixture and in the reaction zone coincide with the corresponding conditions for disturbed flames with the unaltered conditions in the unburned mixture and that the values $M_{\mathcal{A}}$ are effectively equivalent for these two cases (although the propagation speeds are different).

It should be noted that only the flame stretch $K_{f}$ (but not the flame curvature $\Gamma_{f}$ ) is present in the equations utilized in Proposition 1. This conclusion is valid for any reaction mechanism provided the reaction zone remains thin compared to the flame thickness. However, if the width of the reaction zone is comparable to the flame thickness, both of the parameters $K_{f}$ and $\Gamma_{f}$ can be independently present in the propagation speed equation even when the reaction mechanism is represented by a one-step reaction (several examples illustrating this point are given in Klimenko, Class and O'Gorman (2002)). While in the present work the reference flame location is conventionally defined as being within the thin reaction zone, the case of wider reaction zones considered by Klimenko et al. (2002) requires accuracy in the definition of the reference flame location.

In general, the substitutions specified in the proposition result in a quite cumbersome equation for the propagation speed. For example, the assumption that the function $F_{\mathcal{A}}(\ldots)$ is a smooth and uniform function of its arguments results in the following equation

$$
m_{u r}=m_{*}+\varepsilon K_{f}\left(\begin{array}{c}
\left(\frac{\partial F_{\mathcal{A}}}{\partial T_{b}}\right)_{*} \frac{(\Delta h)_{*}}{C_{\mathcal{A} u}\left(c_{p}\right)_{b *} m_{*}}\left[\phi\left(L_{\mathcal{A}}\right)-\phi(1)\right]+ \\
+\sum_{\mathcal{I}}\left(\frac{\partial F_{\mathcal{A}}}{\partial C_{\mathcal{I} b}}\right)_{*} \frac{\left(\Delta C_{\mathcal{I}}\right)_{*}}{C_{\mathcal{A u}} m_{*}}\left[\phi\left(L_{\mathcal{A}}\right)-\phi\left(L_{\mathcal{I}}\right)\right]- \\
-\left[\phi\left(L_{\mathcal{A}}\right)+\phi_{\rho}\right]
\end{array}\right)+O\left(\varepsilon^{2}\right)
$$


where the sum is taken over $\mathcal{I}=\mathcal{B}, \mathcal{P}_{1}, \mathcal{P}_{2}, \ldots$ and $m_{*}=m_{0}$. In this equation, we formally expand the function $F_{\mathcal{A}}(\ldots)$ into a series in powers of $\varepsilon$ and neglect the terms $\partial / \partial \tau$ which appear to be out of order when $m_{u r}=m_{*}+O(\varepsilon)$. Equation (46) is quite large and it is not very convenient for practical purposes since it does not take into account specific properties of the consumption rate function $F_{\mathcal{A}}(\ldots)$. The equation obtained by the method specified in the proposition may be simplified when specific assumptions about the properties of the function $F_{\mathcal{A}}(\ldots)$ are applied.

\section{The large Zeldovich number asymptotes}

Since the reaction rate is high in the reaction zone and negligible in the rest of the flame, we can expect that the rates of some of the reactions should be very sensitive to the value of the temperature (i.e. some of the reactions should have a large activation temperature). Thus, we can expect that the dependence of $M_{\mathcal{A}}=F_{\mathcal{A}}\left(T_{b}, \ldots\right)$ on $T_{b}$ is stronger than the dependence on the other parameters. Mathematically, this can be expressed by introducing an effective Zeldovich number, $Z_{f}$, defined by the dimensionless expression

$$
Z_{f} \equiv 2 z_{f}(\Delta h)_{*}, \quad z_{f} \equiv \frac{1}{M_{\mathcal{A}}} \frac{\partial M_{\mathcal{A}}}{\partial h_{b}}=\frac{1}{\left(c_{p}\right)_{b} M_{\mathcal{A}}} \frac{\partial M_{\mathcal{A}}}{\partial T_{b}}=\frac{1}{\left(c_{p}\right)_{b}} \frac{\partial \ln \left(M_{\mathcal{A}}\right)}{\partial T_{b}}
$$

and assuming that $Z_{f} \gg 1$. The value $z_{f}$ is a dimensional $(\sim 1 / h)$ characteristic response of the reaction zone to a temperature change. The effective Zeldovich number, which involves $(\Delta h)_{*}$ in its definition, is a dimensionless property not only of the reaction zone but also of the preheat zone. In this section we effectively put $M_{\mathcal{A}}=F_{\mathcal{A}}\left(T_{b}\right)$ (by assuming $Z_{f} \gg 1$ ) while the remaining arguments of the consumption rate function are deemed to be constant. For the one-step mechanism, the assumption of large Zeldovich number is equivalent to the assumption of a thin reaction zone. For more complicated reaction mechanisms, these assumptions may be related but, generally, are not identical.

If $Z_{f}$ and $\left(c_{p}\right)_{b}$ can be assumed to be constant for a certain range of $T_{b}$, then, as determined by $(47)$, the variations of $M_{\mathcal{A}}$ can be approximated by

$$
M_{\mathcal{A}}=F_{\mathcal{A}}\left(T_{b}\right)=\left(M_{\mathcal{A}}\right)_{*} \exp \left(\frac{Z_{f}}{2} \frac{\left(c_{p}\right)_{b *}\left(T_{b}-T_{b *}\right)}{(\Delta h)_{*}}\right)
$$

This exponential dependence can be formally obtained for one-step mechanism (which is considered in Section 6.1) by expanding the argument of the exponent into the Taylor series. Peters (1997) demonstrated that the exponential expression can be a good approximation for the propagation speed of some flames with more complex kinetic mechanisms and introduced $Z_{f}$ in (48) as the apparent Zeldovich number. Approximation (48) is used below to obtain more conventional forms for the equations derived in the present work.

\subsection{The differential diffusion limit}

Sivashinsky (1976) introduced the limit $\varepsilon \rightarrow 0, Z_{f} \rightarrow \infty$ so that $\varepsilon Z_{f} \sim 1$ while the Lewis number $L_{\mathcal{A}}$ is bounded away from unity $\left(L_{\mathcal{A}}-1\right) \sim 1$. In this case the changes of $M$ due to small $(\sim \varepsilon)$ changes of $T_{b}$ are of order of unity while the rest of equation (41), whose order is $O(\varepsilon)$, can be neglected $\rho_{u} U_{f}=\rho_{u} U_{f 0}=m_{0}=m_{u r}=F_{\mathcal{A}}\left(T_{b}\right) / C_{\mathcal{A} u}$ (note that $m_{0} \neq m_{*}$ for this limit). We can inverse this function and write

$$
\begin{gathered}
F_{\mathcal{A}}^{-1}\left(\rho_{u} U_{f} C_{\mathcal{A} u}\right)=T_{b *}+\varepsilon \frac{(\Delta h)_{*}}{\left(c_{p}\right)_{b *} m_{0}}\left(K_{f}+\frac{d}{d \tau}\right)\left[\phi\left(L_{\mathcal{A}}\right)-\phi(1)\right]= \\
=T_{b *}-\tilde{D}_{u} \frac{(\Delta h)_{*}}{\left(c_{p}\right)_{b *} U_{f}}\left(\kappa_{f}+\frac{d}{d t}\right)\left[\frac{\Delta I\left(L_{\mathcal{A}}\right)}{U_{f}}\right]
\end{gathered}
$$

Here, we use $h_{b}$ defined by $(38)$ and $\phi(L)$ defined by $(42)$, assume that $\tilde{D}_{u}$ and $\rho_{u}$ are constants and introduce $\Delta I\left(L_{\mathcal{A}}\right) \equiv I(1)-I\left(L_{\mathcal{A}}\right)$. The adiabatic flame temperature is denoted by $T_{b *}$. This equation 
generalizes Sivashinsky's equation (Sivashinsky 1976) for various $F_{\mathcal{A}}\left(T_{b}\right)$. Sivashinsky's equation can be obtained by substituting the exponential form of the function $F_{\mathcal{A}}\left(T_{b}\right)$ specified by (48) into (49)

$$
\begin{gathered}
U_{f}=U_{f *} \exp \left(-\frac{Z_{f}}{2} \frac{\tilde{D}_{u}}{U_{f}}\left(\kappa_{f}+\frac{d}{d t}\right)\left[\frac{\Delta I\left(L_{\mathcal{A}}\right)}{U_{f}}\right]\right)= \\
=U_{f *} \exp \left(-\frac{Z_{f}}{2} \frac{\tilde{D}_{u} \Delta I\left(L_{\mathcal{A}}\right)}{U_{f}^{2}}\left(\kappa_{f}+\frac{d \ln \left(\Delta I\left(L_{\mathcal{A}}\right) / U_{f}\right)}{d t}\right)\right)
\end{gathered}
$$

Generally, equations obtained for the limit of Lewis numbers bounded away from unity represent nonlinear differential equations with respect to the disturbed flame speed $U_{f}$. The flame speed does not assume fixed values for given conditions ahead of the flame, but it requires a finite time to adjust to changing conditions.

\subsection{The near-equidiffusion limit}

In near equidiffusion flames the Lewis-number $L_{\mathcal{A}}$ is close to unity. Matalon and Matkowsky (1982) assumed that $L_{\mathcal{A}}=1+\lambda / Z_{f}$ where $\lambda \sim 1$ and $Z_{f} \rightarrow \infty$. We estimate that $(\Delta h)_{1} \sim 1 / Z_{f}$ and $\left(M-m_{0}\right) \sim \varepsilon$ so that the change of propagation velocity is small and $d / d \tau$ term can be neglected (note that, for this limit, the leading order approximations coincide with the corresponding undisturbed values: $m_{0}=m_{*}, U_{f 0}=U_{f *}, h_{0}=h_{*}$, etc.). Substituting the expansion

$$
M=m_{*}+\varepsilon \frac{\partial M}{\partial h_{b}}(\Delta h)_{1}+\ldots=m_{*}\left(1+\varepsilon \frac{Z_{f}}{2}(\Delta h)_{1} /(\Delta h)_{0}\right)+\ldots
$$

(note the definition of the effective Zeldovich number in (47) and the following representation $M=$ $\left.F_{\mathcal{A}}\left(T_{b}\left(h_{b}\right), \ldots\right) / C_{\mathcal{A} u}\right)$ into equation (41), while taking into account (38), (42) and (43), yields

$$
\frac{U_{f}}{U_{f *}}=\frac{m_{u r}}{m_{*}}=1+\frac{\varepsilon}{m_{*}} K_{f}\left[\frac{\lambda}{2} \phi^{\prime}(1)-\phi(1)-\phi_{\rho}\right]=1+\kappa_{f} \frac{\tilde{D}_{u}}{U_{f *}^{2}}\left[\left(\Delta L_{\mathcal{A}}\right) \frac{Z_{f}}{2} I^{\prime}(1)-I(1)-I_{\rho}\right]
$$

where $\phi^{\prime} \equiv d \phi / d L, I^{\prime} \equiv d I / d L$, and $\Delta L_{\mathcal{A}} \equiv L_{\mathcal{A}}-1$. This equation generalizes the Matalon and Matkowsky equation (Matalon and Matkowsky 1982) for multi-step kinetics but differs from that equation only by the definition of $Z_{f}$. The equation of Matalon and Matkowsky is commonly used (even when $\Delta L$ is not so small) because of its relative simplicity. With the use of the Markstein number Ma, the Karlovitz number Ka and the undisturbed flame thickness $l_{f *}$, equation (51) can be written as

$$
\frac{U_{f}}{U_{f *}}=1-\mathrm{Ma} \cdot \mathrm{Ka}, \quad \mathrm{Ma} \equiv \frac{Z_{f}}{2} \Delta L_{\mathcal{A}} I_{1}+I_{0}, \quad \mathrm{Ka} \equiv \frac{l_{f *}}{U_{f *}} \kappa_{f}, \quad l_{f *} \equiv \frac{\tilde{D}_{u}}{U_{f *}}
$$

where $I_{1} \equiv-I^{\prime}(1), I_{0}=I(1)+I_{\rho}$. These integrals are evaluated in Appendix A.

\section{Analytical evaluation of the effective Zeldovich number for reduced kinetic mechanisms}

In this section, the consumption rate function $F_{A}(\ldots)$ and the effective Zeldovich number $Z_{f}$ are evaluated analytically for simplified chemical kinetics: 1) the one-step irreversible reaction mechanism, 2) the two-step reaction mechanism which was introduced by Zeldovich (1948), and 3) three-step reduced mechanism which was introduced by Peters and Williams (1987) for methane combustion. The first case demonstrate consistency of the present approach with the approaches of Sivashinsky (1976) and Matalon and Matkowsky (1982) who considered the one-step mechanism. For this mechanism, the effective Zeldovich number $Z_{f}$ is virtually identical to the conventional Zeldovich number $Z$ which is defined using the activation temperature of the reaction. In the second case, we demonstrate that the effective Zeldovich number may be not linked to the activation temperature of any the reactions considered. The third 
case repeats the result of Rogg and Peters (1990) while illustrating the efficiency of our approach. The equation for the effective Zeldovich number bears some similarity with the second case.

The analysis of this section is restricted to the reaction zone since this is sufficient to find $F_{A}(\ldots)$ and $Z_{f}$. In the reaction zone, we consider only the leading order terms as specified by the asymptotic equations in (28). For convenience, we replace the characteristic variable $\xi$ of the reaction zone in equation (28) by the physical coordinate $x$ (unless a more specific analysis is required). Effectively, this analysis is conducted for undisturbed planar flames resulting in finding $F_{A}(\ldots)$ and $Z_{f}$ but not the propagation speed of the disturbed flames. The propagation speed of the disturbed flames $U_{f}$ can be easily determined by substituting the found values of $F_{A}(\ldots)$ and $Z_{f}$ into equations (46), (49) and (51) (which are valid under different conditions) or, alternatively, $F_{A}(\ldots)$ can be used to find $U_{f}$ as specified in Proposition 1. In the rest of the present work, we often use the asterisk subscripts for some of the values (namely $T_{b *}$ and $U_{f *}$ ) to emphasize that the analysis is conducted in Sections 6 and 7 for undisturbed flames and it is sufficient to find $F_{A}(\ldots)$ and $Z_{f}$. When the flames are not disturbed by the fluid flow, there is no difference between "asterisked" and "non-asterisked" variables $T_{b *}=T_{b}, U_{f *}=U_{f}$, etc. Note that, for irreversible reactions considered in the present section, $M_{\mathcal{A}}$ does not depend on burned concentrations of products and $\left(C_{\mathcal{P}_{i}}\right)_{b}$ can be excluded from the argument list of the function $F_{A}(\ldots)$. In this section we assume that $c_{p}=$ const.

\subsection{The one-step mechanism}

For a irreversible one-step Arrhenius reaction the equations within the reaction zone are given by

$$
\begin{gathered}
-D_{b} \frac{d T}{d x}=N_{T} W, \quad-\frac{D_{b}}{L_{\mathcal{A}}} \frac{d C_{\mathcal{A}}}{d x}=-W \\
W=A\left(C_{\mathcal{B}}\right)^{\beta}\left(C_{\mathcal{A}}\right)^{\alpha} \exp \left(-\frac{T_{a}}{T}\right)=A_{r}\left(C_{\mathcal{B}}\right)^{\beta}\left(C_{\mathcal{A}}\right)^{\alpha} \exp \left(z\left(T-T_{b *}\right)\right)+\ldots
\end{gathered}
$$

where $z \equiv T_{a} / T_{b *}^{2}$ and $N_{T} \equiv N_{h} / c_{p}=$ const. Conventional integration of equations (53) and (54) (see Clavin (1985), Williams (1985) for details) indicates that $C_{\mathcal{A}}=L_{\mathcal{A}}\left(T_{b *}-T\right) / N_{T}$ and defines the consumption rate function by

$$
M_{\mathcal{A}}=F_{\mathcal{A}}\left(T_{b *},\left(C_{\mathcal{B}}\right)_{b *}\right)=\left(2 \operatorname{Gamma}(\alpha+1) A_{r} D_{b}\left(\frac{1}{z N_{T}}\right)^{\alpha+1} L^{\alpha}\left(C_{\mathcal{B}}\right)_{b *}^{\beta} \exp \left(-\frac{T_{a}}{T_{b *}}\right)\right)^{1 / 2}
$$

Assuming that $A_{r}=A\left(T_{b *}\right) \sim\left(T_{b *}\right)^{\alpha_{A}}$ and $D_{b}=D\left(T_{b *}\right) \sim\left(T_{b *}\right)^{\alpha_{D}}$ we obtain that the effective Zeldovich number

$$
Z_{f} \equiv 2(\Delta T)_{*} \frac{d \ln (M)}{d T_{b *}}=Z+\left(\alpha_{A}+\alpha_{D}+2 \alpha+2\right) \frac{(\Delta T)_{*}}{T_{b *}} \approx Z
$$

is effectively equivalent to the conventional Zeldovich number $Z$ which is defined by

$$
Z \equiv \frac{T_{a}}{T_{b *}^{2}}(\Delta T)_{*}=z(\Delta T)_{*}=\frac{T_{a}}{T_{b *}^{2}} N_{T} C_{\mathcal{A} u}
$$

since $Z \sim 1 / \varepsilon_{r} \gg 1$ when the reaction zone is thin while the term in (56) involving $(\Delta T)_{*} / T_{b *}$ is of order of unity. Note that the leading order of equation (56) is not affected by the parameters $\alpha$ and $\beta$ of reaction rate equation (54). Obviously, equations (55) and (56) combined with equations (49) and (51) are equivalent to the corresponding equations of Sivashinsky (1976) and Matalon and Matkowsky (1982).

\subsection{The two-step Zeldovich-type mechanism}

Zeldovich (1948) introduced a two-step reaction mechanism assuming that the first reaction has a very high activation energy and no heat effect while the second reaction releases heat but its rate does not depend on the temperature. The typical flame structure for this mechanism is shown in Figure 1. The rate 
of the first reaction is very intense only within the very thin activation zone whose characteristic thickness is denoted by $l_{a}$. Linan (1971) and Seshadri and Peters (1983) considered a similar mechanism with the first reaction causing some heat release which is comparable to the heat release of the second reaction. This formulation requires a numerical integration. In the present example we follow the formulation of Zeldovich (no heat release in the first reaction) which is sufficient for our purposes and allows for analytical representation of the flame structure. The full analysis of the reaction zone is presented in the Appendix B where we consider some minor generalizations of the Zeldovich mechanism for different types of the reactions to determine the sensitivity of the effective Zeldovich number with respect to altering the reaction parameters. We can rewrite equations (B16) and (B17), which determine the consumption rate function, in the form

$$
T_{b *}=\frac{T_{a}}{\left(\alpha \ln \left(\Delta T_{r}\right)-2 \ln \left(M_{\mathcal{A}}\right)-z_{1} \Delta T_{r}+B_{1}\right)}, \quad \Delta T_{r}=B_{2}\left(M_{\mathcal{A}}\right)^{\frac{2}{\beta+1}}
$$

where $B_{1}$ and $B_{2}$ are constants whose exact definition in (B16) and (B17) is obvious but not given here since $Z_{f}$ appears not to depend on these values. The meaning of the parameters $\alpha$ and $\beta$ is given in Appendix B. We use the function $T_{b *}=T_{b *}\left(M_{\mathcal{A}}\right)$ instead of $M_{\mathcal{A}}=F_{\mathcal{A}}\left(T_{b *}\right)$ as the most simple analytical representation of the functional dependence of $T_{b *}$ and $M_{\mathcal{A}}$ for this case. Differentiation of equation (58) indicates that

$$
\frac{d T_{b *}}{d M_{\mathcal{A}}}=\frac{T_{b *}^{2}}{T_{a}}\left(\left(z_{1}-\frac{\alpha}{\Delta T_{r}}\right) \frac{d\left(\Delta T_{r}\right)}{d M_{\mathcal{A}}}+\frac{2}{M_{\mathcal{A}}}\right), \quad \frac{d\left(\Delta T_{r}\right)}{d M_{\mathcal{A}}}=\frac{2}{\beta+1} \frac{\Delta T_{r}}{M_{\mathcal{A}}}
$$

Considering the definition of the effective Zeldovich number by (47), we obtain

$$
\frac{1}{Z_{f}} \equiv \frac{1}{2} \frac{M_{\mathcal{A}}}{(\Delta T)_{*}} \frac{\partial T_{b *}}{\partial M_{\mathcal{A}}}=\frac{1}{\beta+1} \frac{\Delta T_{r}}{(\Delta T)_{*}}+\left(1-\frac{\alpha}{\beta+1}\right) \frac{1}{Z_{1}} \approx \frac{1}{\beta+1} \frac{\Delta T_{r}}{(\Delta T)_{*}}
$$

Here we take into account that $\Delta T_{r} /(\Delta T)_{*} \sim l_{r} / l_{f}$ (as it is illustrated in Figure 1) while $1 / Z_{1} \sim l_{a} / l_{f}$ according to equation (B5). Since $l_{a} \ll l_{f}$, the second term can be neglected.

\subsection{The three-step methane combustion mechanism of Peters and Williams}

In this section we evaluate the effective Zeldovich number for the combustion mechanism suggested by Peters and Williams (1987) for methane combustion. The mechanism involves three reactions: (i) methane consumption in a very thin fuel consumption layer, (ii) CO oxidation and (iii) H2 oxidation in a wider (but still thin) reaction zone. For this case, the undisturbed flame propagation speed is determined by two equations (Rogg and Peters 1990)

$$
T\left(x_{0}\right)=F_{T}(\ldots), \quad U_{f *}=B \frac{T_{u}}{T\left(x_{0}\right)}\left(\frac{T_{b *}-T\left(x_{0}\right)}{T_{b *}-T_{u}}\right)^{2}
$$

where $x_{0}$ represents the location of the fuel consumption layer, $F_{T}$ and $B$ are functions of various rate constants. Exact specifications of $F_{T}$ and $B$ (which are given in Rogg and Peters (1990) ) are not needed here. We note only that these parameters do not depend on $T_{b *}$. The first equation determines the temperature in the fuel consumption layer $T\left(x_{0}\right)$, while the second equation uses $T\left(x_{0}\right)$ to specify the undisturbed propagation speed. The second equation in (61) determines that the consumption rate function for this case is

$$
M_{\mathcal{A}}=B \frac{T_{u}}{T\left(x_{0}\right)}\left(\frac{T_{b *}-T\left(x_{0}\right)}{T_{b *}-T_{u}}\right)^{2} \rho_{u} C_{\mathcal{A} u}
$$

Differentiation of (62) yields

$$
\frac{\partial \ln \left(M_{\mathcal{A}}\right)}{\partial T_{b *}}=\frac{2}{\Delta T_{r}}-\frac{2}{(\Delta T)_{*}}+\frac{\partial \ln \left(C_{\mathcal{A} u}\right)}{\partial T_{b *}}=\frac{2}{\Delta T_{r}}-\frac{1}{(\Delta T)_{*}}
$$

where $(\Delta T)_{*}=T_{b *}-T_{u}$ and $\Delta T_{r}$ is defined by equation $\Delta T_{r} \equiv T_{b *}-T\left(x_{0}\right)$ which is similar to the corresponding "two-step definition" of (B17). Here we consider variations of the burning rate $M_{\mathcal{A}}$ and 
the adiabatic temperature $T_{b *}$ induced by changing $C_{\mathcal{A} u}$ while keeping $T_{u}$ constant (alternative ways of changing $T_{b *}$ are discussed in the next section). The derivative $\partial \ln \left(C_{\mathcal{A} u}\right) / \partial T_{b *}=1 /(\Delta T)_{*}$ is determined from the equation $C_{\mathcal{A} u}=\left(T_{b *}-T_{u}\right) / N_{T}$. The effective Zeldovich, which is defined by (47) and corresponds to $(63)$, is given by

$$
Z_{f}=2(\Delta T)_{*} \frac{d \ln (M)}{d T_{b *}}=4 \frac{(\Delta T)_{*}}{\Delta T_{r}}-2 \approx 4 \frac{(\Delta T)_{*}}{\Delta T_{r}}
$$

Here, we take into account that the effective Zeldovich number is expected to be large $Z_{f} \gg 1$ and neglect the second term since $(\Delta T)_{*} / \Delta T_{r} \sim l_{f} / l_{a} \gg 1$. This equation is, effectively, identical to the equation (116) of Rogg and Peters (1990) (if we rewrite that equation using notations of the present work) obtained for planar methane flames. Without claiming any physical similarity of the two-step and three-step cases for $\beta=3$, we note that the value of $Z_{f}$ specified by (64) would be the same as $Z_{f}$ specified by (60) when $\beta=3$ in (B3). The essential common physical feature of the multistep reactions considered above is that the their effective Zeldovich numbers are determined by various reaction rate constants and are not linked to the dimensionless activation temperatures. This feature has been previously noted by Rogg and Peters (1990).

\section{Determining the effective Zeldovich number for realistic flames.}

First, we summarize findings of the previous sections in the following proposition.

Proposition 2 The equation of Matalon and Matkowsky (1982) for propagation speed of a disturbed near-equidiffusion premixed flame with a one-step reaction having a high activation temperature can be used to calculate the propagation speed of a disturbed near-equidiffusion premixed flame with a multi-step kinetic mechanism provided 1) the reaction zone is thin compared to the flame thickness, 2) the effective Zeldovich number $Z_{f}$ is used instead of conventional Zeldovich number $Z$ as specified by equation (51) and 3) the effective Zeldovich number is large. The effective Zeldovich number is defined by equation (47) and can be found from the properties of undisturbed flames. Unlike the conventional Zeldovich number, the effective Zeldovich number is not necessarily linked to the activation temperatures of any of the reaction involved.

The definition of the effective Zeldovich number is based on the consumption rate of the deficient reactant $M_{\mathcal{A}}$. This definition distinguishes rich, lean and stoichiometric (or near-stoichiometric) mixtures. The case of stoichiometric mixtures is most difficult since, depending on conditions in the flow any of the reactants may become deficient when the flame is disturbed. This problem was analyzed by Bechtold and Matalon (1999) for a one-step mechanism and it is not specifically considered in the present work. The effective Zeldovich number can be determined from data on the propagation speed of undisturbed flames. Indeed, since $M_{\mathcal{A}}=C_{\mathcal{A} u} \rho_{u} U_{f *}$, equation (47) can be written as

$$
Z_{f}=2(\Delta T)_{*} \frac{\partial \ln \left(M_{\mathcal{A}}\right)}{\partial T_{b *}}=2(\underbrace{(\Delta T)_{*} \frac{\partial \ln \left(C_{\mathcal{A u}}\right)}{\partial T_{b *}}}_{\text {(I) }}+\underbrace{(\Delta T)_{*} \frac{\partial \ln \left(\rho_{u}\right)}{\partial T_{b *}}}_{(\mathrm{II})}+\underbrace{(\Delta T)_{*} \frac{\partial \ln \left(U_{f *}\right)}{\partial T_{b *}}}_{(\mathrm{III})})
$$

In this section, we assume $c_{p}=$ const and put $(\Delta T)_{*}=(\Delta h)_{*} / c_{p}=T_{b *}-T_{u}$ where $T_{b *}=T_{b}$ since the flame is not disturbed by the flow. The contributions of the terms (I), (II) and (III) are to be assessed now. The temperature of the burned mixture of an undisturbed flame $T_{b *}=T_{u}+N_{T} C_{\mathcal{A} u}$ can be changed by a) altering the temperature of the unburned mixture $T_{u}$ while keeping other parameters constant or b) by changing the unburned concentrations of fuel and oxidizer present in the unburned mixture (that is changing $C_{\mathcal{A} u}$ and keeping $T_{u}$ constant). The choice has an implication for relative significance of the terms in equation (65). If $T_{u}$ is altered while $C_{\mathcal{A} u}$ is kept constant, term (I) is zero while term (II) can be assessed as $-(\Delta T)_{*} \partial \ln \left(T_{u}\right) / \partial T_{b *} \sim\left(-(\Delta T)_{*} / T_{u}\right)$, assuming that $\rho_{u} \sim 1 / T_{u}$. The order of term (II) (can be as large as ten in real flames) is comparable to the order of term (III). If $T_{u}$ is kept constant while $C_{\mathcal{A} u}$ is changed then term (II) is negligible while term (I) takes form $(\Delta T)_{*} /\left(C_{\mathcal{A} u} N_{T}\right)=1$. This value may be neglected if $Z_{f}$ is very large. Method b) also requires that the burned values of the excess reactant 
and the products are kept constant. Since it seems that the concentration of the products should not have a dramatic effect on the flame speed, we require that only $C_{\mathcal{B} b}$ remains constant (i.e. the increments of the unburned values of the reactants are linked by $\left.\Delta C_{\mathcal{B} u}=\left|N_{\mathcal{B}}\right| \Delta C_{\mathcal{A} u}\right)$.

Abdel-Gayed, Bradley, Hamid and Lawes (1984) and Abdel-Gayed et al. (1984a) suggested a method of finding the dimensionless activation temperature $Z_{a} \equiv T_{a} /(\Delta T)_{*}$ using experimental data for the propagation speed. In this method, the activation temperature $T_{a}$ is determined by plotting the available data for $\ln \left(U_{f *}\right)$ versus $1 / T_{b *}$ and defining $T_{a}$ as the doubled negative slope of this curve. One may notice that the dimensionless activation temperature $Z_{a}$ is related to the effective Zeldovich number $Z_{f}$ when $T_{b *} \gg T_{u}$. Indeed, if $T_{a}=2 \partial \ln \left(U_{f *}\right) / \partial\left(1 / T_{b *}\right)$ then $Z_{a} \sim T_{a} / T_{b *}=2 T_{b *} \partial \ln \left(U_{f *}\right) / \partial T_{b *} \sim Z_{f}$. The activation temperatures determined by Abdel-Gayed, Bradley, Hamid and Lawes (1984) and AbdelGayed et al. (1984a) ) do not remain constant and appear to be larger for higher $T_{b *}$. This indicates that, for the realistic hydrogen, methane and propane reactions analyzed by Abdel-Gayed, Bradley, Hamid and Lawes (1984) and Abdel-Gayed et al. (1984a), the values $Z_{a}$ and $Z_{f}$ are not determined by the activation energy of any single reaction. We determine the value of the effective Zeldovich number from experimental data presented by Abdel-Gayed, Bradley, Hamid and Lawes (1984) and Abdel-Gayed et al. (1984a) without using the concept of the activation temperature. The data do not indicate any significant difference between lean and rich mixtures. First, we approximate the dependence of $\ln \left(U_{f *}\right)$ on $T_{b *}$ by the second order polynomial $a_{0}+a_{1} T_{b *}+a_{2} T_{b *}^{2}$ whose coefficients are adjusted to give the best fit to the propagation speed data as shown in Figure 2. It appears that the quadratic term is insignificant for hydrogen and methane flames. The value of the Zeldovich number is then determined as specified by equation (65). Since $T_{u}=328 \mathrm{~K}$ was kept constant in the experiments of Abdel-Gayed, Bradley, Hamid and Lawes (1984) and Abdel-Gayed et al. (1984a) the term (II) of equation (65) is zero. The determined values of the effective Zeldovich number $Z_{f}$ are plotted in Figure 3 . We note that $Z_{f}$ is larger for higher $T_{b *}$ for hydrogen, methane and propane flames considered in the present work. The value of the Zeldovich number $Z$ defined for the one-step mechanism by equation (57) demonstrates the opposite tendency: $Z$ is smaller for higher $T_{b *}$.

The value of the Markstein number defined by equation (52) is shown in Figure 4. The Lewis numbers are assumed constant and taken $L=0.3$ for hydrogen, $L=0.98$ for methane, $L=1.8$ for propane and $L=1.05$ for oxygen. For rich flames, when oxygen is deficient, all of the curves (some of them are not shown) are very close to the dashed line which corresponds to the equidiffusion case $(L=$ 1). Therefore the Markstein number Ma for lean methane flames is very close to the corresponding equidiffusion value. The values of Ma for lean propane flames are much larger than for methane. When the flame is essentially stable (lean methane and propane flames and rich hydrogen flames) the determined values can be compared with the characteristic experimental values for Ma. The minimal values of Markstein number $(\mathrm{Ma} \approx 2.8$ for methane and $\mathrm{Ma} \approx 4.2$ for propane) are consistent with the lean asymptotes of these values $(\mathrm{Ma} \approx 2.7$ for methane and $\mathrm{Ma} \approx 4.4$ for propane) reviewed by Clavin (1985) and determined from experimental data of Quinard, Searby and Boyer (1984) and Searby and Quinard (1990). For rich hydrogen flames, the value $\mathrm{Ma} \approx 5.7$ determined from experiments is noticeably higher than the predicted Ma. The assumption of a thin reaction zone should not work well for the hydrogen flames since, as it has been noted by Dixon-Lewis (1979), in hydrogen flames the reaction zone can be wider than the preheat zone.

\section{Conclusions}

In the present work, we consider the propagation speed of premixed flames with a multi-step reaction mechanism disturbed and curved by an arbitrary and unsteady fluid flow. The reaction zone is assumed to be thin compared to the flame thickness and the flame thickness is assumed to be small compared to the characteristic length scale of the fluid flow. The combustion process involves two reactants (fuel and oxidizer), one or several final products which are present in the burned mixture and several intermediate products (such as radicals) which are present only within the flame. It is also expected that the deficient reactant (denoted by $\mathcal{A}$ ) does not appear in the products. The major results are now summarized.

1) It is shown that if the propagation speed of an undisturbed flame is known (and specified by the function $M_{\mathcal{A}}=F_{\mathcal{A}}(\ldots)$ determining the mass consumption rate of the deficient reactant) for varying conditions in the burned mixture, then the propagation speed of the disturbed flame can be determined as specified in Proposition 1.The propagation speed of premixed flames with complex chemistry depends 
on the flame stretch but, provided the reaction zone is thin compared to the flame thickness, not on the flame curvature (as an independent parameter). The general method can be simplified when specific properties of the function $F_{\mathcal{A}}(\ldots)$ are taken into account.

2) In a practically important case, when the propagation speed of undisturbed flame is most sensitive to the changes of temperature in the burned mixture $T_{b}$, the effective Zeldovich number $Z_{f}$ introduced by equation (47) is expected to be large. Depending on the values of the Lewis number, we distinguish two characteristic limits: the differential diffusion limit which is similar to the limit introduced by Sivashinsky (1976) for one-step mechanisms and the near-equidiffusion limit which is similar to the limit introduced by Matalon and Matkowsky (1982). The near-equidiffusion limit is most often used in applications due to the relatively simple structure of the final equation.

3) The near-equidiffusion equation (51), which was introduced by Matalon and Matkowsky (1982) for the propagation speed of the flames with a one-step reaction mechanism, is shown to be valid for multistep reaction mechanisms provided the conventional Zeldovich number $Z$ is replaced by the effective Zeldovich number $Z_{f}$ (Proposition 2). Information about the propagation speed of undisturbed flames is sufficient to determine the effective Zeldovich number.

4) The effective Zeldovich number is analytically determined for three reaction mechanisms: the onestep irreversible reaction, the two-step mechanism introduced by Zeldovich (1948) and the three-step methane combustion mechanism of Peters and Williams (1987). In the first case, as one can expect, the effective Zeldovich number is almost identical to the conventional Zeldovich number which is linked to the activation temperature (or energy) of the reaction. The second and third examples demonstrate that, under certain conditions, the effective Zeldovich number may be not linked to activation energy of any reaction. The result obtained here for the third example are effectively identical to the results of Rogg and Peters (1990) who considered methane planar flame near a stagnation point and also noted that the conventional Zeldovich number is replaced by a certain combination of reaction constants.

5) The values of the effective Zeldovich number $Z_{f}$ and the Markstein number Ma are determined for hydrogen, methane and propane flames from the experimental data presented by Abdel-Gayed, Bradley, Hamid and Lawes (1984) and Abdel-Gayed et al. (1984a) for the propagation speed of undisturbed premixed flames. The Markstein numbers Ma for lean propane/air and lean methane/air flames are consistent with the experimental data of Quinard et al. (1984) and Searby and Quinard (1990) while the values of Ma for rich hydrogen flames are somewhat underestimated in the present analysis. 


\section{References}

Abdel-Gayed, R. G., Al-Khishali, K. J. and Bradley, D.: 1984a, Turbulent burning velocity and flame straining in explosions, Proc. Roy. Soc. of London A A391, 393-414.

Abdel-Gayed, R. G., Bradley, D., Hamid, M. N. and Lawes, M.: 1984, Lewis number effects on turbulent burning velocity, Twentieth Symposium (Int.) on Combustion, The Combustion Institute, Pittsburgh, $P A$ pp. 505-512.

Bechtold, J. K. and Matalon, M.: 1999, Effects of stoichiometry on stretched premixed flames, Combust. and Flame 119, 217-232.

Berman, V. S. and Riazantsev, I. S.: 1973, Asymptotic analysis of stationary propagation of the front of a two-stage exothermic reaction in a gas, J. Appl. Math. Mech. 37, 995.

Bradley, D., Lau, A. K. C. and Lawes, M.: 1992, Flame stretch rate as a determinant of turbulent burning velocity, Phil. Trans. R. Soc. Lond. A 338, 359-387.

Buckmaster, J. D. and Ludford, G. S. S.: 1983, Lectures on mathematical combustion, SIAM CBMS 43.

Clavin, P.: 1985, Dynamic behavior of premixed flame fronts in laminar and turbulent flows, Prog. Energy Combust. Sci. 11, 1-59.

Darrieus, G.: 1945, Propagation d'un front de flamme. presented at Le Congrés de Mechanique Appliquée, Paris.

Dixon-Lewis, G.: 1979, Kinetic mechanism, structure and properties of premixed flames in hydrogenoxygen-nitrogen mixtures, Trans. Roy. Soc. Lond. 292, 45-99.

Kapila, A. K. and Ludford, G. S. S.: 1977, Two-step sequential reactions for large activation energies, Combust. and Flame 29, 167.

Karlovitz, B., Denniston, D. W., Knapschaefer, H. D. and Wells, F. E.: 1953, Studies on turbulent flames, Fourth Symposium (Int.) on Combustion, The Combustion Institute, Pittsburgh, PA pp. 613-620.

Klimenko, A. Y.: 1998, Examining the cascade hypothesis for turbulent premixed combustion, Combust.Sci. and Tech. 139, 15-40.

Klimenko, A. Y. and Class, A. G.: 2000, On premixed flames as gasdynamic discontinuities: A simple approach to derive their propagation speed, Combust. Sci. and Tech. 160, 25-37.

Klimenko, A. Y., Class, A. G. and O'Gorman, S. M.: 2002, Near-equidiffusion disturbed premixed flames with wider reaction zones, Technical Report 2002/01, Dept. of Mech. Eng., The University of Queensland.

Landau, L. D.: 1944, On the theory of slow combustion, Acta Physiocochimic URSS 19, 77.

Linan, A.: 1971, A theoretical analysis of premixed flame propagation with an isothermal chain reaction, Technical Report Technical Report No. 1, Instituto Necional De Tecnica Aerospacial "Esteban Terradas", Madrid, Spain.

Margolis, S. B. and Matkowsky, B. J.: 1982, Flame propagation with a sequential reaction mechanism, SIAM J. Appl. Math. 42, 1175.

Markstein, G. H.: 1953, Experimental and theoretical studies of flame front stability, J. Aero. Sci. 18, 199.

Matalon, M.: 1983, On flame stretch, Combust. Sci. and Tech. 31, 169-181.

Matalon, M. and Matkowsky, B. J.: 1982, Flames as gasdynamic discontinuities, J. Fluid Mech. 124, 239259.

Peters, N.: 1997, Kinetic foundation of thermal flame theory, in W. Sirignano, A. Merzhanov and L. D. Luca (eds), Advances in combustion science: In honor of Y. B. Zeldovich, Prog. Astronautics and Aeronautics, 173, pp. 73-91. 
Peters, N. and Williams, F. A.: 1987, The asymptotic structure of stoichiometric methane-air flames., Combust. and Flame 68(2), 185-207.

Quinard, J., Searby, G. and Boyer, L.: 1984, Cellular structures on premixed flames in a uniform laminar flow, in J. E. Wesfreid and S. Zaleski (eds), Lecture Notes in Physics 210: Cellular Structures and Instabilities, Springer, Berlin, pp. 331-341.

Rogg, B. and Peters, N.: 1990, The asymptotic structure of weakly strained stoichiometric methane-air flames, Combust. and flame 79, 402-420.

Searby, G. and Quinard, J.: 1990, Direct and indirect measurements of markstein numbers of premixed flames, Combust. and Flame 82(3), 298-311.

Seshadri, K. and Peters, N.: 1983, The influence of stretch on a premixed flame with two-step kinetics, Combust. Sci. and Tech. 33, 35-63.

Sivashinsky, G. I.: 1976, On a distorted flame front as a hydrodynamic discontinuity, Acta Astronautica 3, 889-918.

Smooke, M. D.: 1991, Reduced Kinetic Mechanisms and Asymptotic Approximations for Methane-Air Flames, Vol. 384 of Lecture Notes in Physics, Springer, Berlin Heidelberg.

Williams, F. A.: 1985, Combustion Theory, 2nd edn, Addison-Wesley, Reading, MA.

Zeldovich, Y. B.: 1948, Theory of flame propagation, Zhur. Fizi. Khi. (USSR) 22, 27-49. English Translation in NACA TM 1282 (1951).

Zeldovich, Y. B., Barenblatt, G. I., Librovich, V. B. and Makhviladze, G. M.: 1985, The mathematical theory of combustion and explosions, Consultants Bureau. 


\section{APPENDICES}

\section{A Equation summary and evaluation of integrals for the near- equidiffusion limit.}

In Appendix A, we offer the summary of the equations and the analytical evaluation of the integrals for the near-equidiffusion case with $c_{p}=$ const and constant Lewis numbers. These equations are commonly used in applications due to their relative simplicity

$$
\begin{gathered}
\frac{U_{f}}{U_{f *}}=1-\mathrm{Ma} \cdot \mathrm{Ka} \\
\mathrm{Ma} \equiv \frac{Z_{f}}{2} \Delta L_{\mathcal{A}} I_{1}+I_{0}, \quad \mathrm{Ka} \equiv \frac{l_{f *}}{U_{f *}} \kappa_{f}, \quad Z_{f} \equiv 2(\Delta T)_{*} \frac{\partial \ln \left(U_{f *} \rho_{u} C_{\mathcal{A} u}\right)}{\partial T_{b *}} \\
(\Delta T)_{*} \equiv T_{b *}-T_{u}, \quad l_{f *} \equiv \frac{\tilde{D}_{u}}{U_{f *}}=\frac{D_{u}}{m_{*}}, \quad \Delta L_{\mathcal{A}} \equiv L_{\mathcal{A}}-1, \quad D \equiv \tilde{D} \rho, \quad \widehat{(\ldots)} \equiv \frac{(\ldots)}{(\ldots)_{u}} \\
I_{1} \equiv \int_{T_{u}}^{T_{b *}} \hat{\rho}(T) \hat{D}(T) \ln \left(\frac{(\Delta T)_{*}}{T-T_{u}}\right) \frac{d T}{(\Delta T)_{*}}, \quad I_{0} \equiv \int_{T_{u}}^{T_{b *}} \hat{D}(T)\left(\frac{\hat{\rho}(T)}{(\Delta T)_{*}}+\frac{1-\hat{\rho}(T)}{T-T_{u}}\right) d T
\end{gathered}
$$

The flame stretch $\kappa_{f}$ involves two components: 1)stretch due to divergence of the fluid velocity on the flame surface and 2)the flame curvature. Although equation (A1) determines the propagation speed of disturbed and curved premixed flames, the involved parameters (such as the effective Zeldovich number $Z_{f}$ ) are linked to the structure of corresponding undisturbed and planar flames as indicated by the subscript "*". Assuming $\hat{\rho}(T)=T_{u} / T$ and $\hat{D}(T)=\left(T / T_{u}\right)^{\alpha}$ we note that the theoretical value for $\alpha$ is $\alpha=0.5$ while experimental values lay usually in the range $\alpha \in[0.6,0.7]$ (note that $D=\tilde{D} \rho$ is the "dynamic" diffusion coefficient and that the Mach number is assumed to be small). Integration of $I_{0}$ gives

$$
I_{0}=\frac{1}{\alpha} \frac{r^{2 \alpha}-1}{1-1 / r^{2}}, \quad r \equiv\left(\frac{T_{b *}}{T_{u}}\right)^{1 / 2}
$$

Generally, the integral $I_{1}$ can not always be represented by an analytical expressions. If $\alpha=1$ then $I_{1}=1$. If $\alpha=1 / 2$ then $I_{1}$ also can be integrated

$$
I_{1}=\frac{2}{r^{2}-1}\left(2 r+\ln \left(\frac{r-1}{r+1}\right)-\ln \left(r^{2}-1\right)-2+2 \ln (2)\right), \quad \alpha=\frac{1}{2}
$$

where $r$ is defined in (A5). Linear interpolation of $I_{1}$ between $\alpha=1 / 2$ and $\alpha=1$ can be recommended for a simple approximate evaluation of this integral.

\section{B Analysis of the reaction zone for the two-step Zeldovich-type reaction mechanism}

We consider the two-step reaction mechanism which is governed by equations

$$
\begin{gathered}
-D_{b} \frac{\partial^{2} C_{\mathcal{A}}}{\partial x^{2}}=-W_{1}, \quad-D_{b} \frac{\partial^{2} C_{\mathcal{R}}}{\partial x^{2}}=N_{\mathcal{R}}\left(W_{1}-W_{2}\right), \quad-D_{b} \frac{\partial^{2} T}{\partial x^{2}}=N_{T} W_{2} \\
W_{1}=A_{1}\left(C_{\mathcal{R}}\right)^{\alpha} C_{\mathcal{A}} \exp \left(-\frac{T_{a 1}}{T}\right)=A_{1}\left(C_{\mathcal{R}}\right)^{\alpha} C_{\mathcal{A}} \exp \left(-\frac{T_{a 1}}{T_{b *}}\right) \exp \left(z_{1}\left(T-T_{b *}\right)\right)+\ldots
\end{gathered}
$$




$$
W_{2}=A_{2}\left(C_{\mathcal{R}}\right)^{\beta}
$$

where

$$
z_{1} \equiv \frac{Z_{1}}{(\Delta T)_{*}}=\frac{T_{a 1}}{T_{b *}^{2}}
$$

is used in the conventional expansion of the exponential term within the reaction zone. $\mathcal{A}$ is the deficient reactant, $\mathcal{R}$ is an intermediate product (likely a radical) and $Z_{1}$ is the conventional Zeldovich number of the first reaction. Since $W_{2}$ does not depend on $T$, the activation energy and Zeldovich number of the second reaction are zeros: $T_{a 2}=0$ and $Z_{2}=0$. The deficient reactant is consumed in the first, chain-branching step which generates the intermediate product. The rate of this reaction is $W_{1}$ and its heat effect is negligible. The second step whose rate is given by $W_{2}$ converts the intermediate product into the final products and releases heat. If the parameters of the reactions are selected so that $\alpha=1$ and $\beta=2$, then equations (B1)-(B3) represent the two-step Zeldovich mechanism whose first and second reactions are given by 1) $\mathcal{A}+\mathcal{R} \rightarrow \mathcal{R}+\mathcal{R}$ and 2) $\mathcal{R}+\mathcal{R} \rightarrow \mathcal{P}+$ heat. Since $W_{2}$ does not depend on $T$, the activation energy and Zeldovich number of the second reaction are zeros $T_{a 2}=0$ and $Z_{2}=0$.

Here, we follow, to a large extend, the analysis of Seshadri and Peters (1983), who considered the behavior of the flame with Zeldovich two-step mechanism $(\alpha=1$ and $\beta=2)$ in a stagnation point flow. We assume that the Zeldovich number of the first reaction is sufficiently large so that the consumption of the reactant $\mathcal{A}$ occurs in the activation zone which is thinner than the reaction zone:

$$
l_{a} \sim \frac{l_{f}}{Z_{1}} \ll l_{r}
$$

The heat is released by the second reaction within the reaction zone whose characteristic thickness is $l_{r}$. Unlike in the original Zeldovich's (Zeldovich (1948) ) formulation of the mechanism, Seshadri and Peters (1983) investigate the case when the heating value of the first reaction is not negligible and this requires numerical evaluation of the solution within the activation zone. The formulation of Zeldovich (1948), generalized for arbitrary values of parameters $\alpha$ and $\beta$, is sufficient for our purposes. The flame structure is shown in Fig.1. The rate of the first reaction $W_{1}$ is essential only within the activation zone. If the second reaction is very fast, then the radicals are immediately converted into products, $C_{\mathcal{R}}$ is small and $W_{1} \approx W_{2}$. This case is effectively equivalent to the conventional one-step mechanism and it is not of interest in this section. In the present work we consider the case when the rate $W_{2}$ is sufficiently slow to provide essential concentration of $C_{\mathcal{R}}$ and, at the same time, sufficiently fast to ensure that $l_{r} \ll l_{f}$.

\section{B.1 The activation zone}

The activation zone is analyzed first. Within the activation zone, the temperature and the concentration of $\mathcal{R}$ is represented by the expansions $T-T_{b *}=\theta_{*}+\theta_{1} x+\ldots$ and $C_{\mathcal{R}}=C_{\mathcal{R}}\left(x_{0}\right)+\ldots$ where $x_{0}$ specifies the $x$-location of the activation zone and is close to zero. Indeed, the heat release and consumption of $\mathcal{R}$ occurs in the reaction zone so that $T=T(\xi)$ and $C_{\mathcal{R}}=C_{\mathcal{R}}(\xi)$ where $\xi$ is the characteristic variable of the reaction zone $\xi=\left(x-x_{0}\right) / \varepsilon_{r}$. Since $l_{a} \ll l_{r}$, the characteristic variable of the activation zone is $\tilde{\xi}=\xi / \varepsilon_{a}$ where $\varepsilon_{a} \equiv l_{a} / l_{r}$ is a small parameter. The functions $T\left(\varepsilon_{a} \tilde{\xi}\right)$ and $C_{\mathcal{R}}\left(\varepsilon_{a} \tilde{\xi}\right)$ are expanded into the Taylor series $T=T\left(x_{0}\right)+\varepsilon_{a} T^{\prime}\left(x_{0}\right) \tilde{\xi}+\ldots$ and $C_{\mathcal{R}}=C_{\mathcal{R}}\left(x_{0}\right)+\ldots$ We have to retain more terms in the temperature series (which can be written in the form $T-T_{b *}=\theta_{0}+\theta_{1} x+\ldots$ when $\tilde{\xi}$ is replaced by the physical coordinate $x$ ) since $T$ is multiplied by a large value, $z_{1}$, in the exponential term of (B2). The coefficients $\theta_{0}$ and $\theta_{1}$ are not known and are to be determined by matching with the heat release zone. The first reaction begins when the temperature becomes sufficiently high and terminates when $C_{\mathcal{A}} \rightarrow 0$. The leading order representation of the $C_{\mathcal{A}}$ transport equation (B1) is given by

$$
-\frac{D_{b}}{L_{\mathcal{A}}} \frac{d^{2} C_{\mathcal{A}}}{d x^{2}}=-A_{1}^{*} C_{\mathcal{A}} \exp \left(z_{1} \theta_{0}+z_{1} \theta_{1} x\right), \quad A_{1}^{*} \equiv A_{1}\left(C_{\mathcal{R}}\left(x_{0}\right)\right)^{\alpha} \exp \left(-\frac{T_{a 1}}{T_{b *}}\right)
$$

The solution of (B6), which satisfies $C_{\mathcal{A}} \rightarrow 0$ as $x \rightarrow \infty$, is given by

$$
C_{\mathcal{A}}(x)=M_{\mathcal{A}} \frac{2 L_{\mathcal{A}}}{z_{1} \theta_{1} D_{b}} \operatorname{BesselK}_{0}\left(\frac{2}{z_{1} \theta_{1}}\left(\frac{A_{1}^{*} L_{\mathcal{A}}}{D_{b}}\right)^{1 / 2} \exp \left(\frac{z_{1} \theta_{0}}{2}+\frac{z_{1} \theta_{1}}{2} x\right)\right)
$$


The main characteristics of this solution are given by the integrals

$$
\begin{gathered}
M_{\mathcal{A}} \equiv \int_{-\infty}^{+\infty} W_{1} d x=-\frac{D_{b}}{L_{\mathcal{A}}} \lim _{x \rightarrow-\infty}\left(\frac{d C_{\mathcal{A}}}{d x}\right) \\
x_{0} \equiv \frac{1}{M_{\mathcal{A}}} \int_{-\infty}^{+\infty} x W_{1} d x=\frac{D_{b}}{M_{\mathcal{A}} L_{\mathcal{A}}} \lim _{x \rightarrow-\infty}\left(C_{\mathcal{A}}-x \frac{d C_{\mathcal{A}}}{d x}\right)=\frac{1}{z_{1} \theta_{1}}\left(\ln \left(\frac{z_{1}^{2} \theta_{1}^{2} D_{b}}{A_{1}^{*} L_{\mathcal{A}}}\right)-\gamma_{0}\right)-\frac{\theta_{0}}{\theta_{1}}
\end{gathered}
$$

where $\gamma_{0} \equiv 2 \gamma$ and $\gamma \approx 0.577$ is the Euler constant. Finally, we use equation (B9) and evaluate the leading order of $T$ and $T^{\prime} \equiv d T / d x$ at $x=x_{0}$

$$
T\left(x_{0}\right)-T_{b *}=\theta_{0}+\theta_{1} x_{0}=\frac{1}{z_{1}}\left(\ln \left(\frac{z_{1}^{2} \theta_{1}^{2} D_{b}}{A_{1}^{*} L_{\mathcal{A}}}\right)-\gamma_{0}\right), \quad T^{\prime}\left(x_{0}\right)=\theta_{1}
$$

\section{B.2 The reaction zone}

The reaction zone (or the heat release zone) is now considered. Within the heat release zone, the radicals which are produced in the activation zone diffuse upstream and downstream while they are converted into products and heat. The source of the radicals $\mathcal{R}$ is located somewhere near $x=0$ and can be treated as the delta-function since $l_{a} \ll l_{r}$. A more precise location of the source of $\mathcal{R}$, which increases accuracy of matching, is $x_{0}$ determined by (B9). The leading order equations are given by

$$
-\frac{D_{b}}{L_{\mathcal{R}}} \frac{d^{2} C_{\mathcal{R}}}{d x^{2}}=\left(M_{\mathcal{A}} \delta\left(x-x_{0}\right)-\left(C_{\mathcal{R}}\right)^{\beta} A_{2}\right) N_{\mathcal{R}}, \quad-D_{b} \frac{d^{2} T}{d x^{2}}=\left(C_{\mathcal{R}}\right)^{\beta} A_{2} N_{T}
$$

The boundary conditions for these equations are given by $C_{\mathcal{R}} \rightarrow 0$ as $x \rightarrow \pm \infty$ and $T \rightarrow T_{b *}$ as $x \rightarrow \infty$. Integration of (B11) for $x>x_{0}$ yields

$$
\frac{D_{b}}{2 L_{\mathcal{R}}}\left(\frac{d C_{\mathcal{R}}}{d x}\right)^{2}=\frac{A_{2} N_{\mathcal{R}}}{\beta+1}\left(C_{\mathcal{R}}\right)^{\beta+1} \text { and } T=T_{b *}-\frac{N_{T}}{N_{\mathcal{R}} L_{\mathcal{R}}} C_{\mathcal{R}} \text { for } x>x_{0}
$$

The delta-function term in (B11) determines the value of the derivative of $C_{\mathcal{R}}$ at $x=x_{0}$ (note that $C_{\mathcal{R}}$ is symmetric with respect to the point $x=x_{0}$ ) so that

$$
\begin{gathered}
\left(\frac{d C_{\mathcal{R}}}{d x}\right)_{x=x_{0}}=\frac{L_{\mathcal{R}}}{2 D_{b}} M_{\mathcal{A}} N_{\mathcal{R}}, \quad C_{\mathcal{R}}\left(x_{0}\right)=\left(\frac{\beta+1}{8} \frac{L_{\mathcal{R}}}{D_{b} A_{2}} M_{\mathcal{A}}^{2} N_{\mathcal{R}}\right)^{\frac{1}{\beta+1}} \\
T^{\prime}\left(x_{0}\right)=\left(\frac{d T}{d x}\right)_{x=x_{0}}=\frac{M_{\mathcal{A}} N_{T}}{2 D_{b}}, \quad T_{b *}-T\left(x_{0}\right)=\frac{N_{T}}{L_{\mathcal{R}} N_{\mathcal{R}}}\left(\frac{\beta+1}{8} \frac{L_{\mathcal{R}}}{D_{b} A_{2}} M_{\mathcal{A}}^{2} N_{\mathcal{R}}\right)^{\frac{1}{\beta+1}}
\end{gathered}
$$

\section{B.3 Asymptotic matching}

Matching of $T^{\prime}\left(x_{0}\right)$ and $T\left(x_{0}\right)$ specified by equations (B10) and (B13) yields

$$
\begin{gathered}
\frac{M_{\mathcal{A}} N_{T}}{2 D_{b}}=\theta_{1} \\
\frac{1}{z_{1}}\left(\ln \left(\frac{A_{1}^{*} L_{\mathcal{A}}}{z_{1}^{2} \theta_{1}^{2} D_{b}}\right)+\gamma_{0}\right)=-\frac{1}{z_{1}} \frac{T_{a 1}}{T_{b *}}+\frac{1}{z_{1}}\left(\ln \left(\frac{A_{1}\left(C_{\mathcal{R}}\left(x_{0}\right)\right)^{\alpha} L_{\mathcal{A}}}{z_{1}^{2} \theta_{1}^{2} D_{b}}\right)+\gamma_{0}\right)
\end{gathered}
$$

Equations (B14, B13, B12, B4, and B15) define the functional dependence $T_{b *}=T_{b *}\left(M_{\mathcal{A}}, \Delta T_{r}\left(M_{\mathcal{A}}\right)\right)$ by the formulae

$$
\begin{gathered}
T_{b *}=\frac{T_{a}}{\ln \left(\frac{4 D_{b} A_{1}\left(L_{\mathcal{R}} N_{\mathcal{R}} \Delta T_{r}\right)^{\alpha} L_{\mathcal{A}}}{z_{1}^{2} M_{\mathcal{A}}^{2} N_{T}^{2-\alpha}}\right)-z_{1} \Delta T_{r}+\gamma_{0}} \\
\Delta T_{r} \equiv T_{b *}-T\left(x_{0}\right)=\frac{N_{T}}{L_{\mathcal{R}} N_{\mathcal{R}}}\left(\frac{\beta+1}{8} \frac{L_{\mathcal{R}}}{D_{b} A_{2}} M_{\mathcal{A}}^{2} N_{\mathcal{R}}\right)^{\frac{1}{\beta+1}}
\end{gathered}
$$

which implicitly determine $M_{\mathcal{A}}$ as a function of $T_{b *}$. The physical meaning of the temperature increment $\Delta T_{r} \equiv T_{b *}-T\left(x_{0}\right)$ is demonstrated in Figure 1 . 


\section{FIGURE CAPTURES}

Figure 1. Schematic of the flame structure for the Zeldovich two-step reaction mechanism

Figure 2. The propagation speed of undisturbed premixed flames versus the temperature of the burned mixture. The data are taken from Abdel-Gayed, Bradley, Hamid and Lawes (1984) and Abdel-Gayed et al. (1984a) . Small symbols correspond to experimental points while the large symbols mark the ends of the approximating curves. Solid line - the fit by a second order polynomial.

Figure 3. The values of the effective Zeldovich number $Z_{f}$ versus temperature of the burned mixture. The approximations $U_{f *}\left(T_{b *}\right)$ and notations are taken from Figure 2.

Figure 4. The value of the Markstein number calculated for lean flames (solid lines) and rich flames (dashed line). The dash-dotted line shows the value of Ma for equidiffusion case $(L=1)$. 


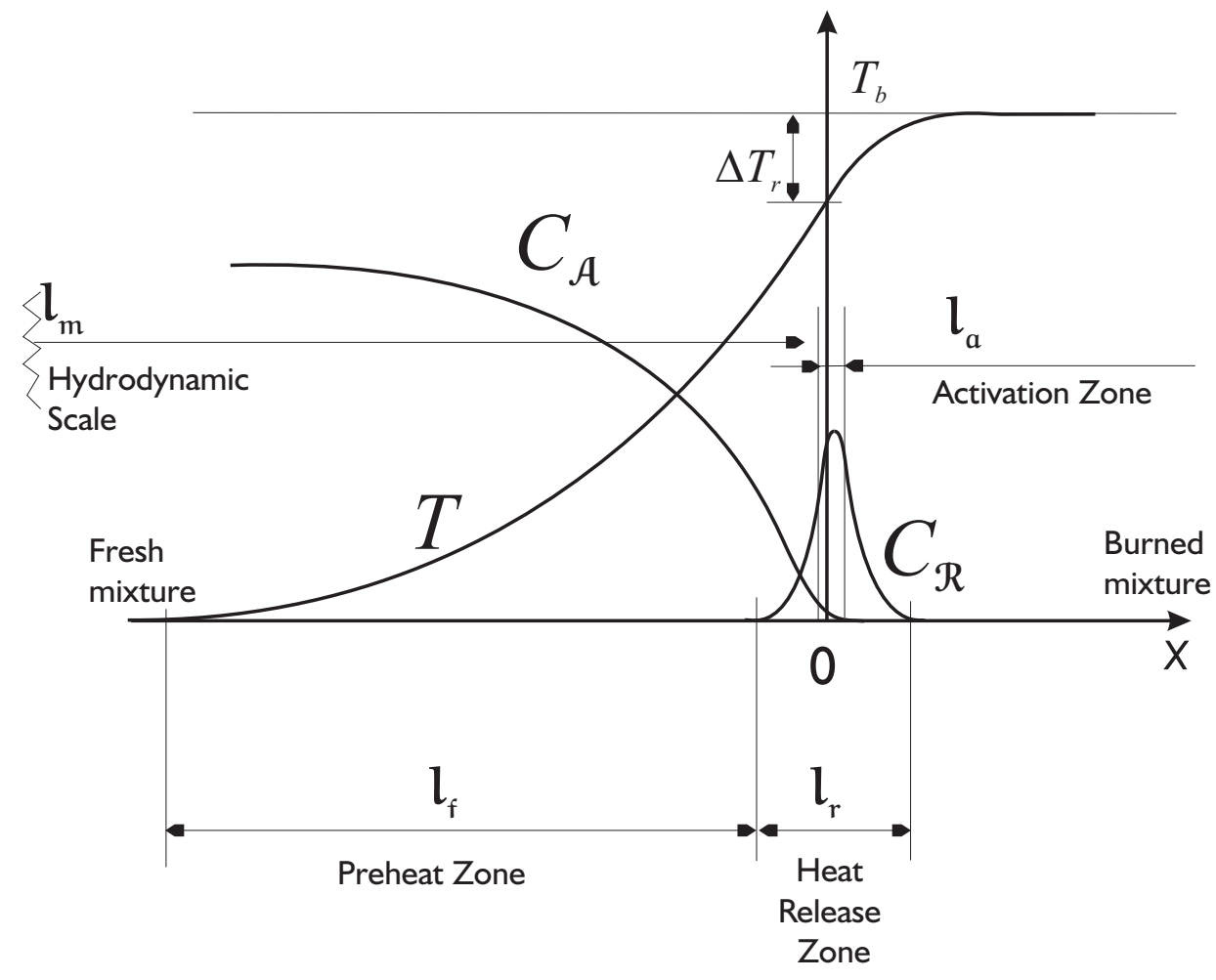

Fig. I 


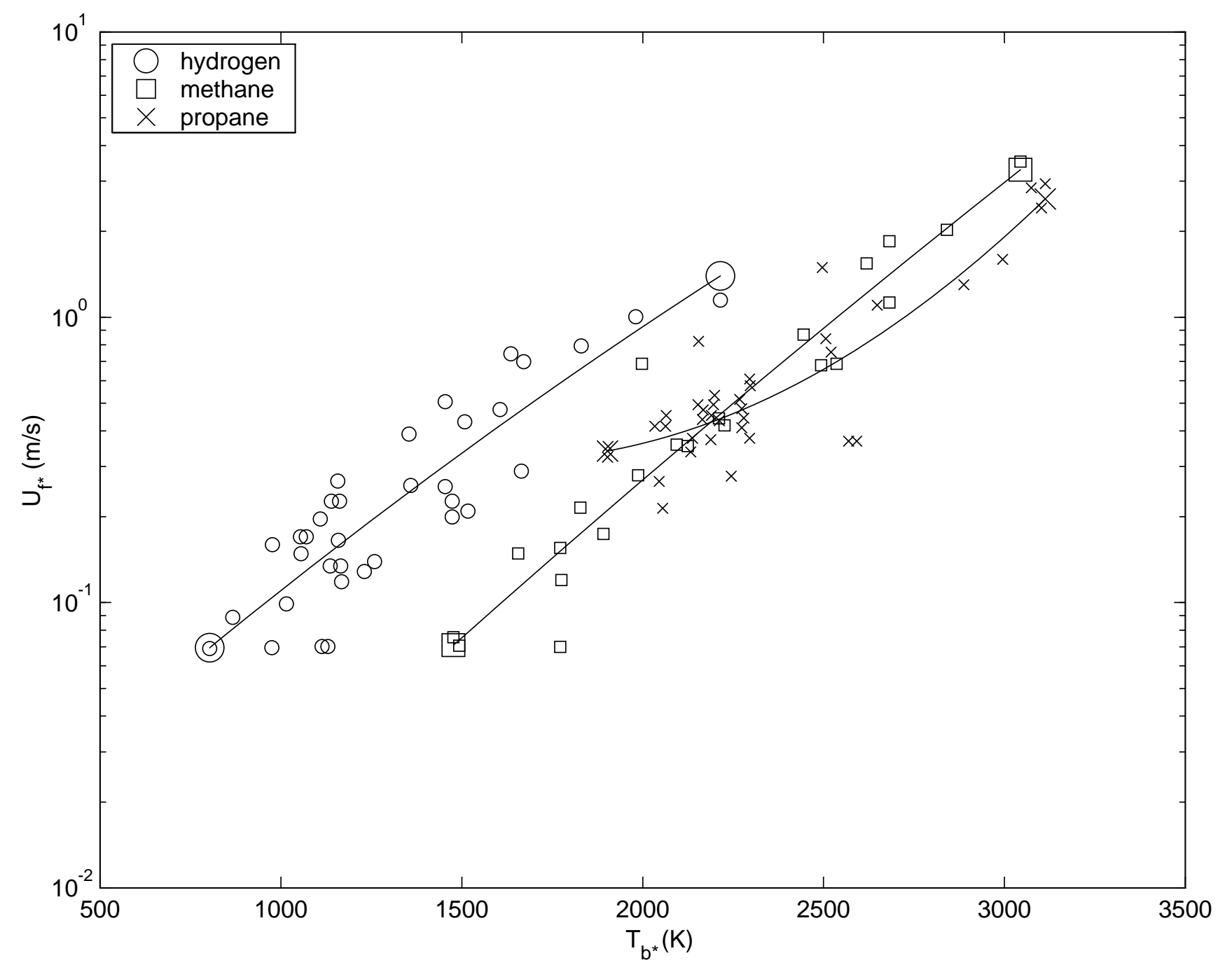

Fig.2 


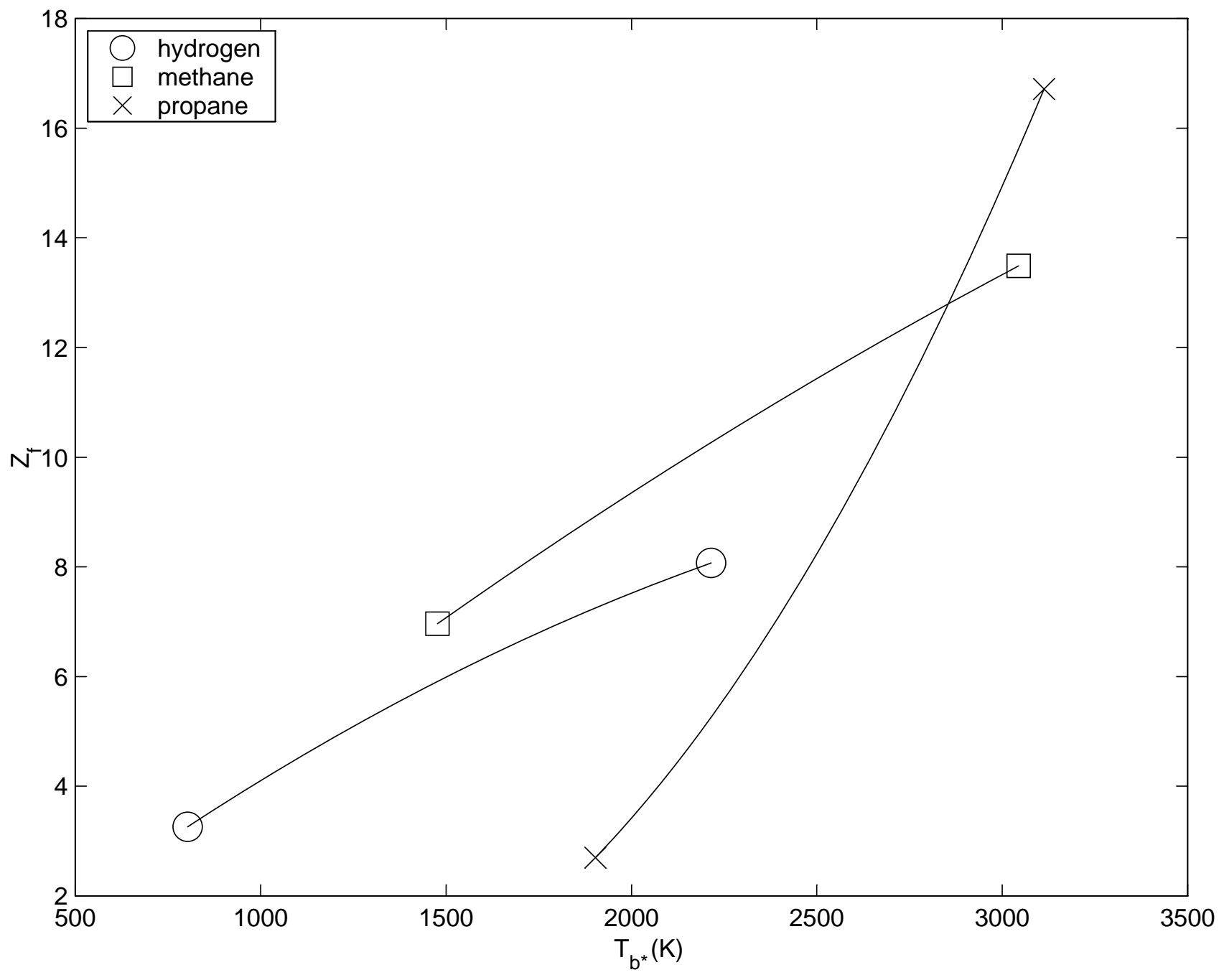

Fig. 3 


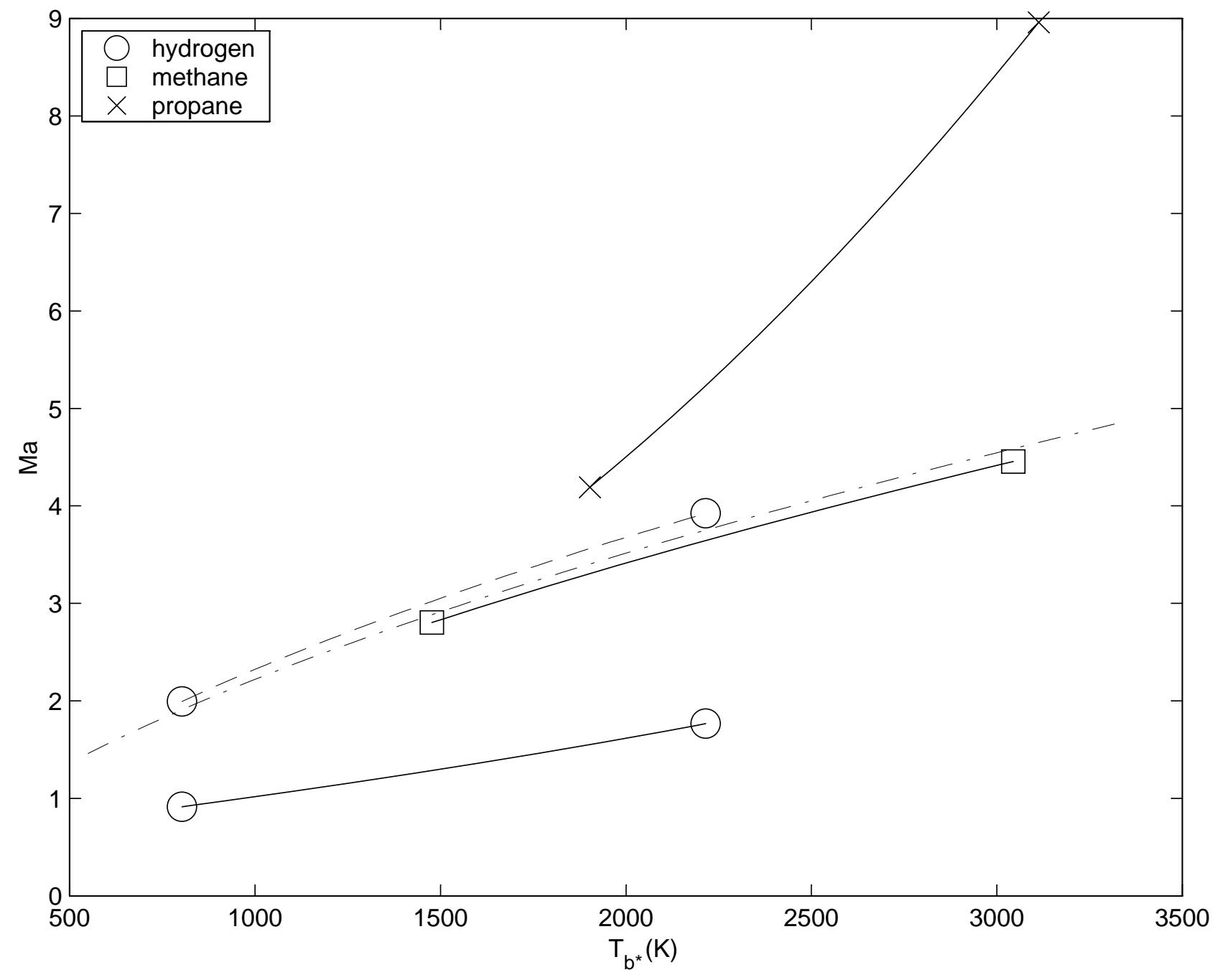

Fig.4 